CONFORMAL GEOMETRY AND DYNAMICS

An Electronic Journal of the American Mathematical Society

Volume 1, Pages 58-86 (November 14, 1997)

S $1088-4173(97) 00014-3$

\title{
A "REGULAR" PENTAGONAL TILING OF THE PLANE
}

\author{
PHILIP L. BOWERS AND KENNETH STEPHENSON
}

\begin{abstract}
The paper introduces conformal tilings, wherein tiles have specified conformal shapes. The principal example involves conformally regular pentagons which tile the plane in a pattern generated by a subdivision rule. Combinatorial symmetries imply rigid conformal symmetries, which in turn illustrate a new type of tiling self-similarity. In parallel with the conformal tilings, the paper develops discrete tilings based on circle packings. These faithfully reflect the key features of the theory and provide the tiling illustrations of the paper. Moreover, it is shown that under refinement the discrete tiles converge to their true conformal shapes, shapes for which no other approximation techniques are known. The paper concludes with some further examples which may contribute to the study of tilings and shinglings being carried forward by Cannon, Floyd, and Parry.
\end{abstract}

\section{INTRODUCTION}

First, of course, there is no regular pentagonal tiling of the plane! That is to say, no euclidean one. However, our tilings will be regular in a "conformal" sense, a notion which we have not seen in the literature. We could generate many examples, but as for the particular tiling $\mathcal{T}$ which will occupy center stage here, let us not prolong the suspense: it is illustrated in Figure 1.

The motivation for looking at this particular tiling pattern lies in work of J. Cannon, W. Floyd, and W. Parry, and others in the context of subdivision rules for shinglings of the plane - ongoing work in the study of hyperbolic groups and efforts towards a proof of Thurston's Geometrization Conjecture. (See [7,6, 12].) Our pentagonal case is in many ways a toy problem in these developments; nonetheless, its intricacies eluded the authors until recent work on related triangular structures. We are treating it for its intrinsic beauty, for new mapping techniques it has engendered, and for questions it raises about combinatorial structures. The value of these ideas for more involved patterns remains to be seen, but for readers familiar with work on subdivision rules, we summarize pertinent connections at the end of the paper.

Our construction of $\mathcal{T}$ involves classical, infinite, completely abstract processes. Coupled with the fact that conformal structures are notoriously difficult to pin down in the best of circumstances, it is a pleasant surprise to find that we can approximate the conformal data for $\mathcal{T}$ using circle packings. In Figure 2 we display a circle packing associated with our tiling combinatorics; if you connect the centers of tangent shaded circles, you will see a (polygonal) tiling in the pattern of $\mathcal{T}$ emerge.

Received by the editors April 28, 1997 and, in revised form, August 21, 1997.

1991 Mathematics Subject Classification. Primary 05B45, 30C30; Secondary 30F20.

Key words and phrases. Tiling, subdivision rules, circle packing, conformal maps.

The second author gratefully acknowledges support of the National Science Foundation and the Tennessee Science Alliance. 


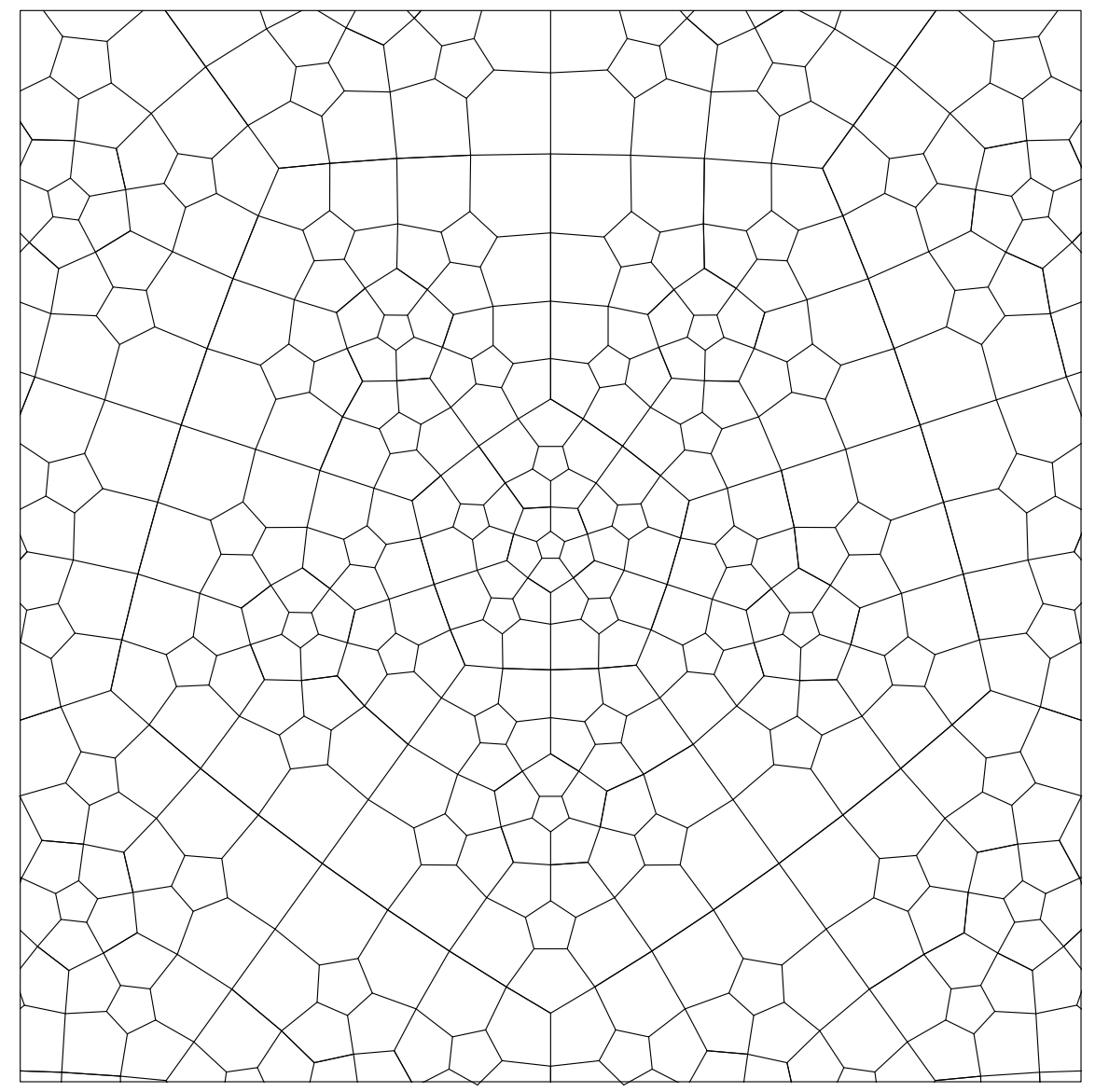

FiguRe 1. A conformally regular pentagonal tiling $\mathcal{T}$ of the plane.

However, our circle packings do more than mimic $\mathcal{T}$. As a sort of half-way house between purely combinatoric and rigidly conformal structures, they provide new insights of their own. Moreover, we introduce techniques for refining the packings to obtain shapes which converge to the conformal tiles. For instance, Figure 3 displays approximations to three representative tiles from $\mathcal{T}$. To our knowledge, there are no classical mapping techniques for such approximations.

The paper has four parts. We construct the tiling of Figure 1 in Part I and discuss its properties, both globally and in terms of individual tile shapes. Of potential interest are two packing constants that arise in the construction. In Part II we work with associated circle packings to obtain additional global information as well as concrete approximations to the tiles. We introduce the necessary background on circle packings and quasiconformal mappings. In Part III we consider generalizations to other tile patterns. Finally, we return to the work which inspired this study in Part IV.

The authors thank Bill Floyd and Walter Parry for valuable conversations on the material presented here. Our illustrations were created using CirclePack, a package written by the second author, with data provided by Bill Floyd. 


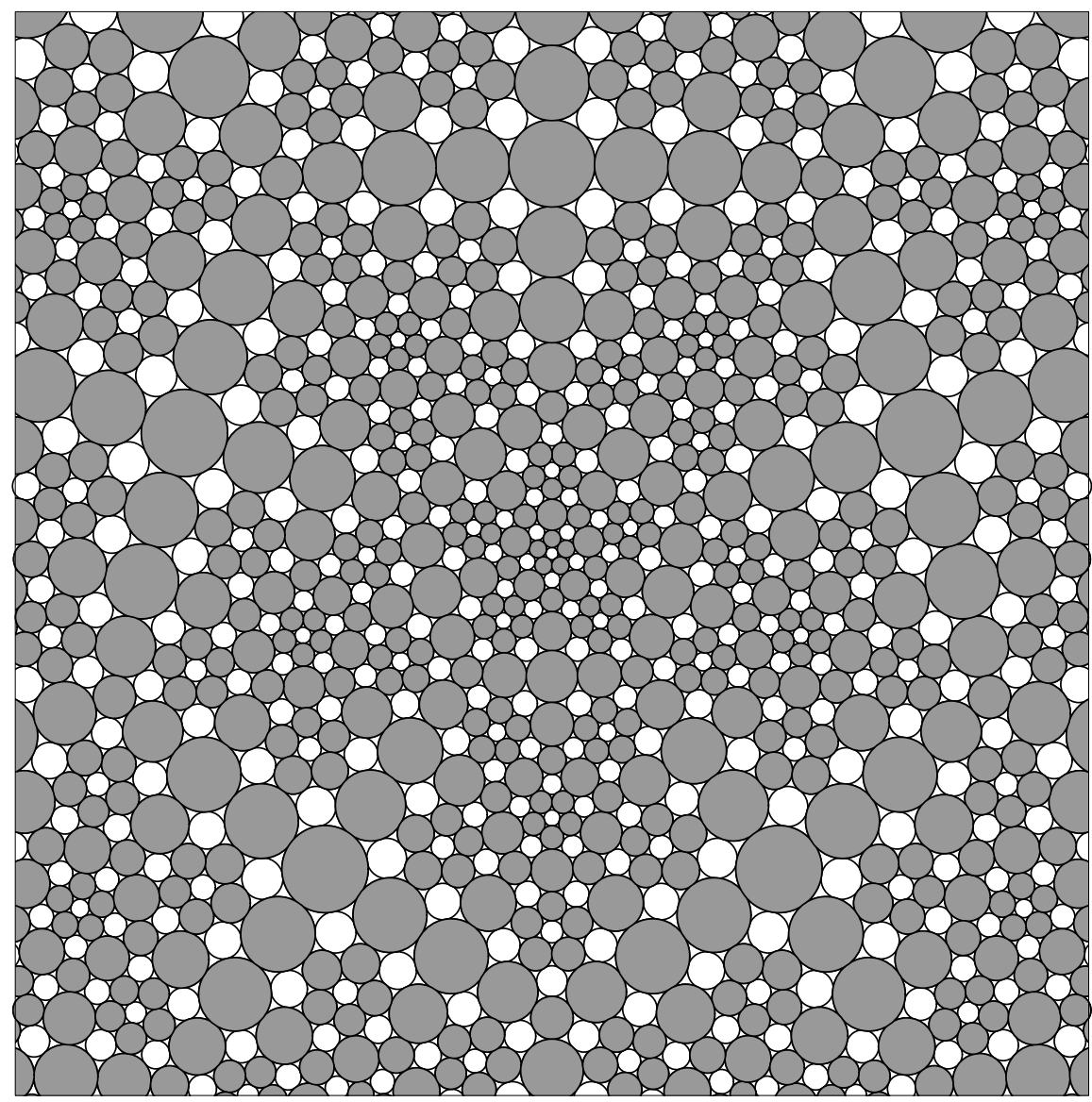

FIGURE 2. The "maximal packing" associated with $\mathcal{T}$.
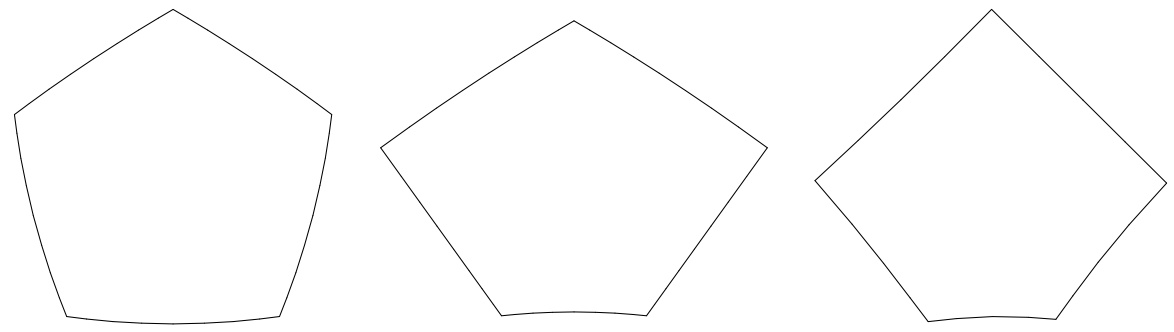

FiguRE 3. Three prototype tiles.

\section{Part I. The Conformally Regular Tiling}

The objects we manipulate in building our tiling are hardly exotic — in fact, they are easy to visualize and will be familiar enough to the reader that we dispense with most technical details. Some knowledge of conformal structures and the Riemann 
Mapping Theorem would be helpful; see, for example, Ahlfors/Sario [1] or Beardon $[2]$.

A brief overview may be useful to highlight the way that these familiar objects evolve as we move from an abstract encoding of the pattern through layers of progressively more rigid structures, each new layer being compatible with its predecessors.

By combinatorics we mean the abstract pattern of tiles - which tiles are next to which others. This will be encoded in an abstract cell complex $K$. It is convenient to include a topology, so each 2-cell or face $f$ is a topological pentagon; that is, a topological closed disc with five distinguished boundary points as corners (or vertices) determining five boundary edges. Each edge is shared with a neighboring face, each corner is shared by a finite number of other faces (in our case, 3 or 4 ). Next, we define a metric on $K$ which induces the original topology while making each cell isometrically isomorphic to a regular euclidean pentagon of side length one. This determines an (singular) affine structure for $K$. In turn, the affine structure determines in a canonical way a compatible conformal structure, making $K$ a Riemann surface. Finally, through the good graces of the Riemann Mapping Theorem, $K$ is mapped conformally onto either a disc or the complex plane. The original cell complex is thereby realized as a tiling, and the final task is to show that it fills the plane rather than a disc.

Note that a face $f$, starting life as an abstract cell of $K$, will successively become a topological pentagon, a regular affine pentagon, a regular conformal pentagon, and finally emerge as a concrete pentagonal tile in the plane. We will abuse notation and use the same symbol $f$ for this face throughout its various incarnations, until it becomes a tile $\tau$. There are certain operations also - local reflections, group actions, isomorphisms - which begin at the combinatorial stage but also respect each new structure; we abuse notation here, too, by sticking with the same names.

\section{The Combinatorial Structure}

The pattern of our tiling is sufficiently straightforward that we can largely avoid the machinery of abstract cell complexes. For us, $K$ is a topological open disc which is decomposed into faces (closed topological pentagons), edges (simple topological arcs), and vertices (points).

We describe $K$ in two ways, starting with the "subdivision" rule which motivated this work. The rule is illustrated in Figure 4 by the transition from $K_{0}$ to $K_{1}$; namely, a combinatorial pentagon $K_{0}$ is divided into six combinatorial pentagons in a certain pattern, yielding $K_{1}$. The further transition to $K_{2}$ results by applying the same rule to each of these six pentagons, giving 36 pentagons. Repeating this ad infinitum, we obtain an infinite collection $\left\{K_{j}\right\}$ of finite pentagonal cell complexes. Note that $K_{0}$ can be identified with the central pentagon of $K_{1}$, that $K_{1}$ can, by matching central pentagons, be identified with a subcomplex of $K_{2}$, and so forth. That is, we have a nested family of cell complexes. $K$ is defined as the directed limit $\lim _{j}=K$ (or union) of the family. Remember that these all represent abstract cell complexes - no geometry is yet imposed.

Alternately, and better for our purposes, construct $K$ as follows: Start again with $K_{0}$. Reflect it (combinatorially) across each of its five edges to obtain a "flower" of six pentagons - $K_{0}$ at the center and five "petal" pentagons. Visit each corner $v$ of $K_{0}$ and paste (i.e., identify) the edges of the two petals which are incident to $v$. 


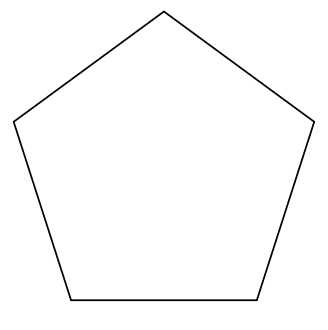

$\mathrm{K}_{0}$

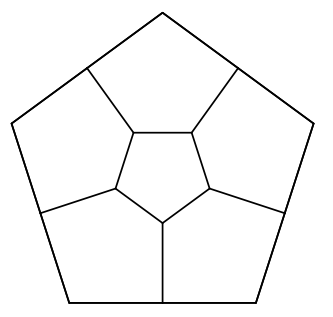

$\mathrm{K}_{1}$

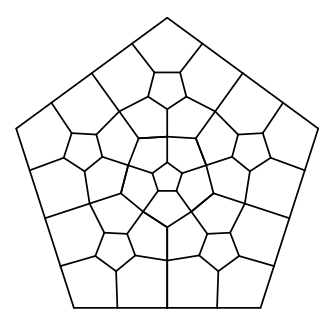

$\mathrm{K}_{2}$

Figure 4. Three stages of $K$.

The result is combinatorially just the complex $K_{1}$ again. A little terminology: the vertices of $\partial K_{1}$ that are incident to only one of the six pentagons forming $K_{1}$ will be called free vertices and become the designated "corners" of $K_{1}$; there are five of them and they break $\partial K_{1}$ into five "edges". Now, we repeat the process with $K_{1}$ : reflect a copy of $K_{1}$ across each of its five edges, carrying its subdivisions with it into each reflected copy. Visit each of the five free corners of $K_{1}$ and paste the boundary edges in the two incident reflected copies. The result is $K_{2}$, which again has precisely five free vertices as corners, breaking its boundary into five edges. Thus we are in position to repeat the process to get $K_{3}$. Iterating ad infinitum, we again arrive at $K$. Since the $K_{n}$ are nested, simply connected complexes, it is evident that $K$ is an infinite pentagonal cell complex homeomorphic to the plane.

The combinatorics of $K$ are intriguing and lie at the heart of our work. The base face of $K$, acting as seed in our constructions, will be denoted by $f_{0}$ and one of its corners by $v_{0}$. It is evident that $K$ has order 5 dihedral symmetry: the group of combinatorial automorphisms, denoted Aut $(K)$, is generated by an order five rotation fixing $f_{0}$ and a reflection fixing $f_{0}$ and $v_{0}$. Treating $K$ as a topological space, the elements of $\operatorname{Aut}(K)$ are homeomorphisms of $K$ with itself, mapping faces to faces, edges to edges, and vertices to vertices. That is, our topological structures are compatible with the underlying combinatorics. A particularly important observation concerns a more subtle form of self-similarity within $K$ : If $K^{\prime}$ is the cell complex obtained from $K$ by applying the pentagonal subdivision rule simultaneously to all its faces, then $K^{\prime}$ is combinatorially equivalent to $K$. Moreover, any combinatorial equivalence must carry the base face of $K^{\prime}$ to the base face of $K$.

There are also important local symmetries. For each edge $e$ of $K$ there are two faces $f_{ \pm}$incident to $e$; let $U_{e}=f_{+} \cup e \cup f_{-}$. We will use $r_{e}$ to denote the local reflection defined on $U_{e}$ by interchanging $f_{+}$with $f_{-}$while fixing $e$. Topologically, treat $e$ as an open edge and $f_{ \pm}$as open faces, so $U_{e}$ is an open set; $r_{e}$ is then an order two orientation-reversing homeomorphism of $U_{e}$.

Finally, we will use a notion of tile aggregation, the reverse of tile subdivision. In particular, we define an infinite family of aggregate faces associated to each face $f$ of $K$. Fix $n \geq 0$ and let $K^{(n)}$ denote the cell complex obtained from $K$ by successively applying the subdivision rule $n$ times. The face $f$ is thereby decomposed into $6^{n}$ faces of $K^{(n)}$. Now by our earlier observation, $K^{(n)}$ is combinatorially equivalent to $K$, so these faces are identified with $6^{n}$ faces back in $K$ itself. The union of these latter faces of $K$ will be called the $n$-stage aggregate face associated with 
$f$, denoted $f^{(n)}$. We emphasize that it is a union of faces of $K$ and that (except in the case of $\left.f=f_{0}\right) f$ is not contained in any $f^{(n)}$. Examples are illustrated in Figure 5: one tile is shown with three of its aggregates (dark shading) and one with one aggregate (light shading). For each $n$, the $n$-stage aggregate faces clearly

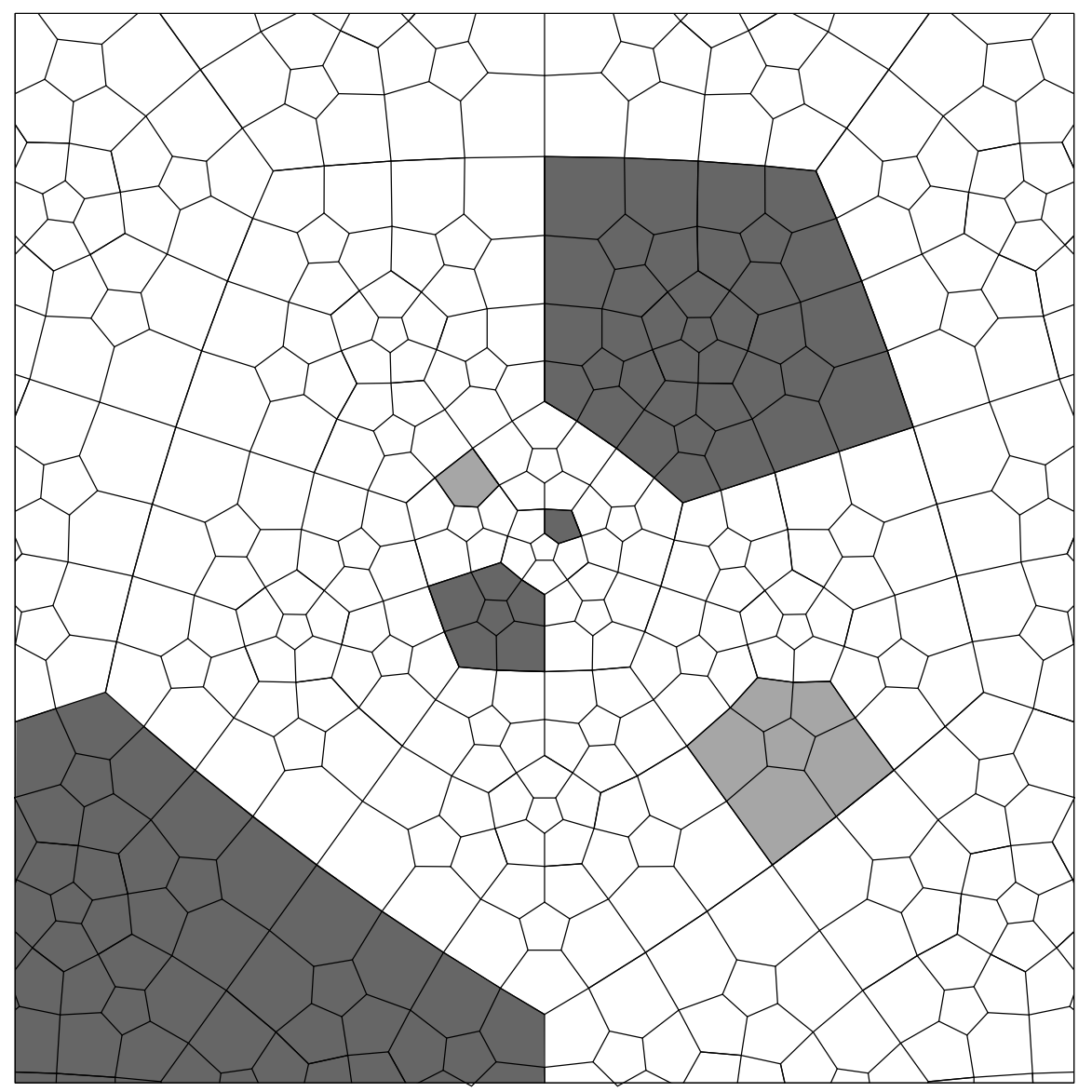

Figure 5. Examples of tile aggregates.

share the same combinatorics; namely, those of $K_{n}$. Each stage of aggregation is the inverse of a stage of subdivision. In parallel with our earlier observation, note that the totality of $n$-stage aggregate faces is combinatorially equivalent to $\mathrm{K}$.

\section{The Affine Structure}

At this point we have combinatorial and topological structures on $K$, but geometry is our central concern. We may realize $K$ as a piecewise affine geometric cell complex as follows: First define the unit edge metric on the one-skeleton of $K$, making each edge isometric to the unit interval. Extend the metric to faces so that each face is isometrically a regular euclidean pentagon (of edge length one). Finally define the distance between an arbitrary pair of points $x, y \in K$ as the length of the shortest path between them. The resulting metric defines the original topology 
on $K$, ensuring compatability with all previous structures. We refer to this metric structure as our affine structure on $K$. (This is equivalent to pasting regular unit pentagons together in the pattern of $K$.)

It is evident that the maps of $\operatorname{Aut}(K)$ are isometries in the affine structure on $K$. The local reflection $r_{e}$ for each edge $e$ is an orientation-reversing isometry of $U_{e}$ onto itself, a local isometry. It is important to note that $r_{e}$ is localized to $U_{e}$ and does not necessarily extend to an isometry on any larger portion of $K$. We may realize $r_{e}$ concretely: Fix two regular euclidean pentagons $P_{1}, P_{2}$ of edge length one in the plane so that they intersect in a common edge $E$ and let $\Omega$ denote the interior of $P_{1} \cup P_{2}$. For each edge $e \in K$, the associated open set $U_{e}$ is isometric to $\Omega$ via a map $\phi_{e}: U_{e} \longrightarrow \Omega$ with $\phi_{e}(e)=E . r_{e}$ is clearly conjugate under $\phi_{e}$ with the euclidean reflection in the line containing $E$ which interchanges $P_{1}$ and $P_{2}$.

\section{The Conformal Structure}

It is standard that a polygonal piecewise affine structure on a surface also determines a unique conformal structure. Recall that a conformal structure will be determined by an atlas $\mathcal{A}=\left\{\left(U_{j}, \phi_{j}\right): j \in J\right\}$ for some indexing set $J$, where each chart $\left(U_{j}, \phi_{j}\right)$ consists of an open set $U_{j} \subset K$ and a homeomorphism $\phi_{j}$ mapping $U_{j}$ to an open set $V_{j}$ in the plane. It is required that the $U_{j}$ cover $K$ and, when $U_{j}$ and $U_{i}$ intersect, that the transition map $\phi_{j} \circ \phi_{i}^{-1}: \phi_{i}\left(U_{j} \cap U_{i}\right) \longrightarrow V_{j}$ be analytic.

We may explicitly define such an atlas for $K$. Let $V$ denote the set of vertices of $K$ and let $\Omega$ be as above. For each edge $e$, the pair $\left(U_{e}, \phi_{e}\right)$ defines a chart. In fact, since each $\phi_{e}: U_{e} \longrightarrow \Omega$ is an isometry, a transition map for two charts is an orientation-preserving isometry mapping some open subset of $\Omega$ back to $\Omega$. In particular, it must be the restriction of a complex linear map $z \mapsto a z+b$, and hence is analytic.

We need only define compatible charts covering $V$. For $v \in V$, let $U_{v}$ be the open metric ball of radius $1 / 3$ centered at $v$. The map $\phi_{v}$ is essentially a power map $z \mapsto z^{p}$ on $U_{v}$, where $p=10 / 3 d$ with $d$ the degree of $v$. Even readers familiar with Riemann surfaces may not have seen this process, so we describe it briefly; for details we recommend Beardon $[2, \S 3.3]$. There is a cycle $f_{1}, f_{2}, \cdots, f_{d}$ of faces incident to $v$. Each $f_{j}$ may be identified isometrically with a regular pentagon in $\mathbf{C}$ with $v$ placed at the origin. The map $\phi_{v}$ is defined on $f_{j} \cap U_{v}$ as $z \mapsto c_{j} z^{p}$, for some unimodular constant $c_{j}$. Set $c_{1}=1$ and successively choose $c_{j}$, to ensure compatibility of $\phi_{v}$ across the edge shared by $f_{j}$ and $f_{j-1}, j=2, \cdots, d$. In our affine structure, each $f_{j}$ has angle $3 \pi / 5$ at $v$. Therefore, if $p \cdot d \cdot 3 \pi / 5$ is an integral multiple of $2 \pi, \phi_{v}$ will be well defined on the edge shared by $f_{1}$ and $f_{d}$. Finally, choosing $p$ so that $p \cdot d \cdot 3 \pi / 5=2 \pi$ ensures that $\phi_{v}$ is one-to-one on $U_{v}$. This gives our chart $\left(U_{v}, \phi_{v}\right)$. Since $U_{v}$ does not intersect $U_{w}$ for any other vertices $w$, one need only check compatibility of this new chart with charts $\left(U_{e}, \phi_{e}\right)$ for edges $e$ emanating from $v$. But this is clear since the power maps are analytic and have nonvanishing derivative away from $v$.

It is easily verified that the mappings of $\operatorname{Aut}(K)$ are now (conformal or anticonformal) automorphisms of the Riemann surface $K$. Moreover, the local edge reflections are anticonformal on their domains. 


\section{The Regular Tiling}

With its conformal structure, $K$ is a simply connected and noncompact Riemann surface. By the Uniformization Theorem, $K$ is conformally equivalent to $D$, where $D$ is either the unit disc $\mathbf{D}$ or the complex plane $\mathbf{C}$. That is, there exists a conformal homeomorphism (i.e., an analytic bijection) $\Phi: K \longrightarrow D$. The images of the faces of $K$ under $\Phi$ form a tiling of $D$ which we now investigate.

First, concerning our notation. We have made it a practice to maintain our notations as we have added layers of structure. This becomes rather counterintuitive, however, with regard to the tiles themselves ( $a k a$, faces): thus we write $\tau$ for a typical concrete tile in $D$, so $\tau=\Phi(f)$ for some face $f$ of $K$, and we will write $\mathcal{T}$ for the collection of tiles, the tiling. The base tile is $\tau_{0}=\Phi\left(f_{0}\right)$. For other notations, we continue our abuse; thus an edge $e \in K$ may refer now to an edge of a tile. Likewise, mappings of $K$ are transplanted to $D$ via conjugation by $\Phi$, so $\psi \in \operatorname{Aut}(K)$ (technically of the form $\Phi \circ \psi \circ \Phi^{-1}$ ) is now a conformal automorphism, hence a Möbius transformation, of $D$ which preserves the tiling $\mathcal{T}$.

Let us first look into the properties of individual tiles. Each $\tau \in \mathcal{T}$ is conformally equivalent under $\Phi$ to some face $f$ of $K$, which in turn is conformally equivalent, in fact isometric (under the charts defining the conformal structure on $K$ ), to a regular euclidean pentagon. Formally, these maps are conformal only on the interiors of these sets and extend continuously to bijections on their closures. So we are treating $\tau$ as a closed set, with the images of the vertices and edges of $f$ providing its vertices and edges.

We refer to $\tau$ as a conformally regular pentagon. Intrinsically, this reflects the fact that $\tau$ admits an order five conformal automorphism - a conformal rotation - and an anticonformal involution which respect the set of vertices. That is, $\tau$ has dihedral symmetry in the conformal sense. There is a unique common fixed point $c$ for these rotations and flips termed the conformal center of $\tau$. From $c$, each edge looks conformally the same.

A local reflection $r_{e}$ in an edge $e$ of $f$ conjugates under $\Phi$ to a local reflection $\Phi \circ r_{e} \circ \Phi^{-1}$ (again called $r_{e}$ ) mapping $\tau$ anticonformally onto a neighboring tile. In particular, each edge $e$ of $\tau$, as a fixed set under $r_{e}$, is an analytic arc in $D$. Note that the five corner angles of $\tau$ are not necessarily equal. In fact, the angle at each corner is determined by the degree of the corresponding vertex $v$ of $K$; if $v$ has degree $d$, then the angle formed at $v$ in $\tau$ (and in the other tiles meeting at $v$ ) will be $2 \pi / d$, as can be seen from the form of the local coordinate at $v$ in the conformal atlas of $K$. Of course, without this angle variability (missing, for example, in regular euclidean polygons) there could be no pentagonal tiling.

A remarkably strong uniqueness result holds for the tile shapes. Indeed, the complete combinatorial structure of $K$ is hard-coded in the shape of each and every tile. Start with a single tile $\tau$, for example. Each edge $e$ is an analytic arc, and the associated anticonformal local reflection $r_{e}$ maps $\tau$ to the neighboring tile across $e$. The edges of that tile are likewise analytic arcs with further local reflections defined on them. By repeated reflections in edges, the original tile $\tau$ will generate the entire tiling $\mathcal{T}$. Suppose tiles $\tau_{1}, \tau_{2}$ were (euclideanly) similar in $D$ and let $f_{1}, f_{2}$ be the corresponding faces of $K$. The tilings generated by anticonformal edge reflections starting from $\tau_{1}$ and from $\tau_{2}$ would be similar, hence combinatorially equivalent. This easily implies existence of a combinatorial automorphism of $K$ mapping $f_{1}$ to $f_{2}$. Thus two tiles of $\mathcal{T}$ are similar in $D$ only if their faces are in the same 
equivalence class of $K$ modulo Aut $(K)$. With our normalization, which places the center of the base tile at the origin, the converse also holds.

We are finally in position to prove that in fact $\mathcal{T}$ tiles $\mathbf{C}$ rather than $\mathbf{D}$. This is a classical "type" problem: from a description of a simply connected open Riemann surface, determine whether that surface is conformally the plane or the disc.

Here we require the underlying symmetry encoded in the combinatorics of $K$. Specifically, we will show, from the fact that $K$ is combinatorially the same as its own pentagonal subdivision $K^{\prime}$, that $D$ admits a conformal automorphism $\alpha$ so that $\alpha^{-n}(z) \longrightarrow 0$ for each $z \in D$. The unit disc (hyperbolic plane) admits no such automorphism, and we must conclude that $\Phi(K)$ tiles the complex plane.

Let us first represent the subdivision rule conformally. Suppose $P$ is a combinatorial pentagon broken into six pentagons by the pentagonal subdivision rule. As with $K$, we can define an affine structure and thereby a conformal structure on $P$ so the six pentagons are conformally regular with local anticonformal reflections across shared edges. With the conformal structure, $P$ is conformally equivalent to the unit disc D. The five-fold symmetry gives an order five conformal automorphism cyclically shifting the outer pentagons. Putting the conformal center of the central pentagon at the origin, we obtain Figure 6; note the corners of $P$ marked on the unit circle. In that Figure, each pair of pentagonal pieces sharing an edge are anticonformal images of one another across the edge.

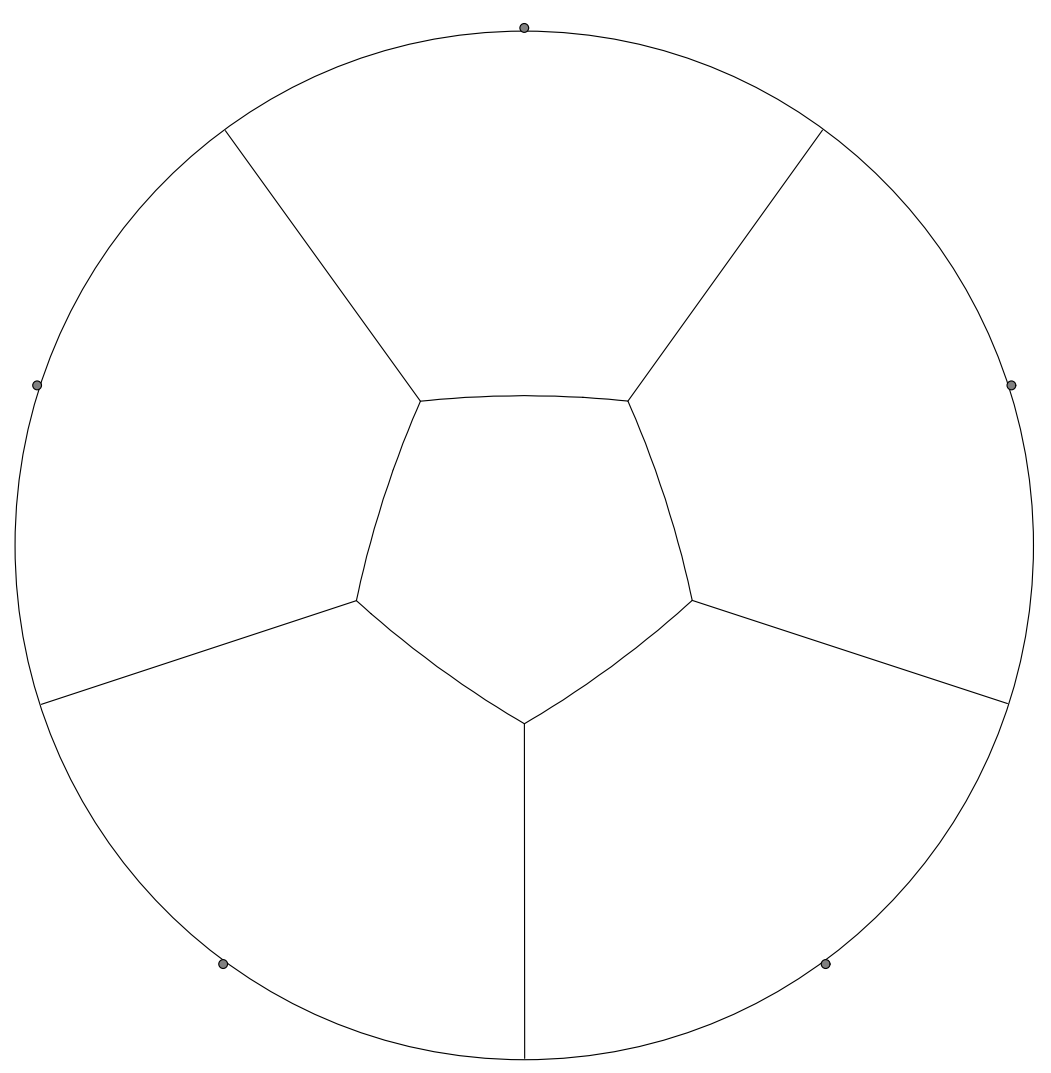

Figure 6 . The conformally correct subdivision rule. 
We now revisit our tiling $\mathcal{T}$ of $D$. Since the base tile $\tau_{0}$ is a conformally regular pentagon, there is a conformal homeomorphism of the unit disc in Figure 6 onto $\tau_{0}$ which maps the corners of $P$ to the corners of $\tau_{0}$. The conformal subdivision of $P$ is therefore carried to a conformal subdivision of $\tau_{0}$. Using the local conformal reflections $r_{e}$, this pattern propagates to give subtilings of all the tiles of $\mathcal{T}$; each subtile is a local reflection of its neighbors within its parent tile, but also of any contiguous subtile in a contiguous parent. The new tiling of $D$ will be denoted $\mathcal{T}^{\prime}$, with $\tau_{0}^{\prime}$ denoting the central tile in $\tau_{0}$.

It is evident that $\mathcal{T}^{\prime}$ has the combinatorics of $K^{\prime}$. As we noted, there is a combinatorial isomorphism from $K^{\prime}$ onto $K$ as cell complexes, hence a conformal mapping of $K^{\prime}$ onto $K$ as Riemann surfaces, hence a conformal mapping of $\mathcal{T}^{\prime}$ onto $\mathcal{T}$ mapping tiles to tiles. In particular, this gives a conformal automorphism $\alpha$ of $D$, which from combinatoric considerations must map $\tau_{0}^{\prime}$ onto $\tau_{0}$ preserving conformal centers. With our normalizations, $\alpha$ fixes $z=0$. Since $\tau_{0}^{\prime}$ lies in a compact subset of $\tau_{0},\left|\frac{d \alpha}{d z}(0)\right|>1$. This implies $\alpha$ is loxodromic, so $D$ can only be the plane, and we have completed the construction of $\mathcal{T}$.

\section{Tiling Constants and Families of Tiles}

The mapping $\alpha$ is a Möbius implementation of the self-similarity we first observed in the combinatorics of $K$. We now see, for instance, that an individual tile $\tau$ is euclideanly similar (precisely!) to the union of tiles forming each of its aggregates. The scaling factor is a characteristic constant associated with $\mathcal{T}$ which the eye quickly picks out in Figure 1. In particular, the automorphism $\alpha$ has the form $\alpha(z)=a \cdot z$ where $\arg (a)=\pi / 5$ and $|a|>1$. From Figure 1 , one can estimate that $|a| \approx 3.2$.

Another characteristic constant $b$ emerges if one looks at more subtle properties of the shapes. Recall that two tiles of $\mathcal{T}$ are (euclideanly) similar if and only if they are equivalent modulo $\operatorname{Aut}(K)$. Thus $\mathcal{T}$ contains an infinite variety of distinct tile shapes. However, each tile $\tau$ is the progenitor of a tile family resulting from the reflective process. Specifically, if $k$ is the smallest integer such that $\tau$ is in the $k$-stage aggregate of $f_{0}$, then for each $n>k$ there are five reflected copies of $\tau$ in $K_{n} \backslash K_{n-1}$, denoted $\tau_{j}^{n}, 1 \leq j \leq 5$. Write $\mathcal{F}_{\tau}=\left\{\tau_{j}^{n}: 1 \leq j \leq 5, n \geq k+1\right\}$. Note that $\tau \notin \mathcal{F}_{\tau}$, every tile is the progenitor of a family, and tile families are mutually disjoint. In a sense, a tile $\tau_{j}^{n} \in \mathcal{F}_{\tau}$ which begins looking around at its combinatoric position will think that it is situated (up to orientation) precisely like $\tau$; only when its inquiries reach out about $(n-k) / 2$ reflective stages will it suddenly find that it is not situated like $\tau$. The conformal tiles are not fooled, but they will give $\tau_{j}^{n}$ a shape more nearly that of $\tau$ as $n$ grows. We make this precise with the following.

Proposition 5.1. There exists a universal constant $b>0$ such that for every $\tau \in \mathcal{T}$ the tiles of $\mathcal{F}_{\tau}$ are asymptotically congruent to $b \cdot \bar{\tau}$.

Here $\bar{\tau}$ denotes the complex conjugate (i.e., flip) of $\tau$. Asymptotically congruent means the following: if $\bar{\tau}$ and each tile of $\mathcal{F}_{\tau}$ are carried by euclidean rigid motions so that each has its conformal center at the origin and a designated corner on the positive $y$-axis, then the tiles $\tau_{j}^{n}$ converge in Hausdorff metric to $b \cdot \bar{\tau}$ as $n$ goes to infinity. The Hausdorff distance between compact sets $X$ and $Y$ in the plane is 
given by

$$
\operatorname{glb}\left\{\delta: \inf _{z \in X}\{|z-y|\}<\delta, \inf _{z \in Y}\{|x-z|\}<\delta, x \in X, y \in Y\right\} .
$$

Proof. The proof depends on the self-similarity transformation $\alpha$ and its relationship to the edge reflections $r_{e}$ in edges of $\tau_{0}$. In particular, there is an $n$-stage local conformal reflection $r_{e}^{(n)}$ which reflects the $n$-stage aggregate face $f_{0}^{(n)}$ to a contiguous $n$-stage aggregate face; it can be defined as follows:

$$
r_{e}^{(n)}(z)=\left(\alpha^{n} \circ r_{e} \circ \alpha^{-n}\right)(z) .
$$

Moreover, rotational symmetry implies that for two edges $e_{1}$ and $e_{2}$ of $f_{0}$,

$$
r_{e_{1}}^{(n)}(z)=(1 / \omega) \cdot r_{e_{2}}^{(n)}(\omega z),
$$

for some fifth root of unity $\omega$.

Fix $\tau \in f_{0}^{(k)} \backslash f_{0}^{(k-1)}$. Given any tile $\tau_{j}^{n}$ in $\mathcal{F}_{\tau}, n>k$, for an appropriate edge $e$ of $f_{0}$,

$$
\tau_{j}^{n}=r_{e}^{(n-1)}(\tau)
$$

By the equalities noted above,

$$
\left|\frac{d r_{e}^{(n)}}{d z}(z)\right| \longrightarrow\left|\frac{d r_{e}}{d z}(0)\right| \text { uniformly for } z \in \tau .
$$

Defining $b=\left|r_{e}^{\prime}(0)\right|$, our result follows.

Thurston and others have studied a more restrictive notion of "self-similarity" and the associated constants (see [20]). Does our constant share any of the surprising properties of theirs? Can one show, for example, whether they are algebraic? Later we will obtain the estimate $b \approx 1.3$ from our circle packings.

\section{Part II. The Circle Packing Tiling}

The construction of $\mathcal{T}$ was completely abstract. Here we show that circle packing provides a comprehensive discrete analogue of the process - with appropriate software, one can actually see global structures emerging in stages. This would be of only passing interest, except that the circle packings can also answer crucial questions about the tiling itself, such as type, and provide computable conformal shapes and estimates for tiling constants such as $a$ and $b$ above. Indeed, all conformal illustrations in the paper, as in Figures 1, 3, and 6, were obtained from circle packings.

We begin with background material on circle packing and on quasiconformal mapping, then describe the discrete construction, and finally show how refinements lead to shape approximations.

\section{BaCkground Material}

Our hope is that later developments can be followed largely through basic geometric intuition and the illustrations. Those not familiar with circle packing and/or quasiconformal mapping can skim this material, but will need to refer to the literature for more depth. 
6.1. Circle Packing Basics. A circle packing is a configuration $P$ of circles having a specified pattern of tangencies. The key to working with a packing is to recognize its separate combinatoric and geometric natures.

The pattern of tangencies for a packing is encoded in a simplicial 2-complex $S$, which is assumed to triangulate a topological surface. A configuration $P$ of circles in some geometry is a circle packing for $S$ if it contains a circle $C_{v}$ associated with each vertex $v$ of $S$ so that circles $C_{v}$ and $C_{u}$ are tangent whenever $\langle u, v\rangle$ is an edge of $S$. Throughout our work here, complexes $S$ will represent infinite triangulations of topological open discs or finite triangulations of topological closed discs; circle packings will lie in the euclidean plane and will be univalent - that is, the circles of a packing will have mutually disjoint interiors.

The geometry of a packing resides with its collection $\mathcal{R}$ of (euclidean) radii, called a label for $S$. Of course, there are stringent compatibility conditions for radii if the circles are to fit together in the plane. (These conditions are most easily expressed in terms of certain "angle sums" at the individual circles.) A label $\mathcal{R}$ meeting the compatibility conditions is called a packing label; using it one can successively lay out the circles with the tangencies prescribed by $S$, and the resulting configuration $P$ is determined up to rigid motions of the plane. The carrier of $P$, denoted $\operatorname{carr}(P)$, is the geometric 2-complex formed by putting a vertex at the center of each circle and connecting the centers of tangent circles with segments. The carrier is an embedding of the abstract complex $S$ in the plane.

The focus in the theory of circle packing is on the existence, variety, and uniqueness of packing labels with given combinatorics and on the geometric properties of their associated packings. In other words, if you are given a pattern $S$, do there exist circle packings $P$ for $S$ satisfying certain specified properties? What are the geometric relationships between distinct packings sharing the same combinatorics? And so forth. The focus in the practice of circle packing is on the computation of packing labels satisfying prescribed conditions and the generation of the associated circle packings - all approximate, of course.

Regarding the combinatorics, our complexes $S$ will be simply connected. When $S$ is finite (i.e., has finitely many vertices), then it triangulates a closed topological disc. Boundary vertices are those which are topologically on the boundary, the rest being interior. When $S$ is infinite, we assume it triangulates an open topological disc; all vertices are interior. The fundamental existence result originated with (independently) Koebe, Andreev, and Thurston (see [5] and references therein).

Discrete Riemann Mapping Theorem. Given a complex $S$ as described above, there exists a univalent circle packing $\mathcal{P}_{S}$ for $S$ called the maximal circle packing which satisfies the following conditions:

(a) If $S$ is finite, then $\mathcal{P}_{S}$ lies in the unit disc $\mathbf{D}$ and has boundary circles which are internally tangent to $\partial \mathbf{D}$.

(b) If $S$ is infinite, then either carr $\mathcal{P}_{S}=\mathbf{D}$ or $\operatorname{carr} \mathcal{P}_{S}=\mathbf{C}$.

The maximal packing $\mathcal{P}_{S}$ is determined uniquely up to conformal automorphisms of $\mathbf{D}$ or $\mathbf{C}$, as appropriate.

The combinatorics of $S$ determine the geometry in which this maximal packing lives, either the euclidean geometry of $\mathbf{C}$ or the hyperbolic geometry of $\mathbf{D}$. In the former case, we say $S$ is parabolic, and in the latter case, hyperbolic. It is known that a parabolic complex $S$ cannot "be packed" in a bounded region of the 
plane (i.e., there exists no packing $P$ for $S$ whose carrier is bounded). Likewise, a hyperbolic complex has no univalent packing filling $\mathbf{C}$.

There are many other existence results for circle packings which we will invoke as necessary. To illustrate with a pertinent example, consider the finite complex $S$ of Figure 7(a). If one randomly designates five of its boundary vertices, then (under minimal technical conditions) there exists a univalent circle packing $P$ for $S$ such that $\operatorname{carr}(P)$ is a pentagon with the designated circles centered at its corners and having interior angles $3 \pi / 5$. See Figure $7(\mathrm{~b})$. Furthermore, the packing $P$ is unique up to Möbius transformations of the plane.

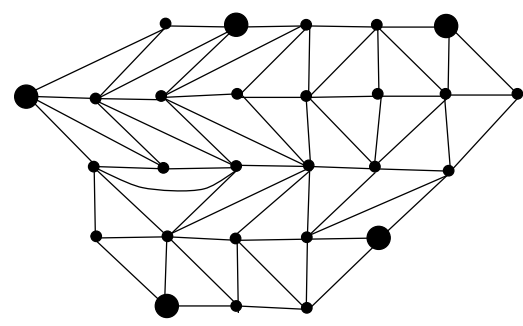

(a)

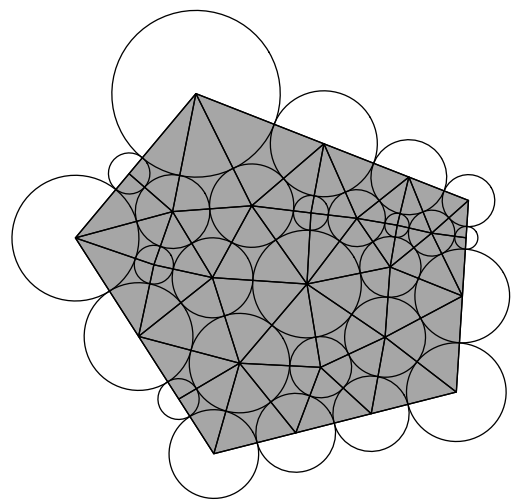

(b)

FiguRE 7. Packing a pentagon.

Next in importance is the issue of geometric properties of packings. If two packings $P$ and $Q$ have the same underlying combinatorics, encoded in $S$, say, then there is a one-to-one correspondence between their sets of circles. We might consider a map $F: P \longrightarrow Q$ which carries each circle of $P$ to the corresponding circle of $Q$. Such mappings are in many ways rather faithful discrete analogues of classical analytic maps; see in particular [8].

However, for the concrete purposes here, proceed as follows: If $P$ and $Q$ are two circle packings for the same complex $S$ (finite or infinite), define the circle packing map $F: P \longrightarrow Q$ by specifying that the center of each circle of $P$ is mapped to the center of its companion in $Q$ and by then extending affinely to the edges and faces of $\operatorname{carr}(P)$. In other words, $F$ is actually a mapping between the two carriers in $\mathbf{C}$ which maps each triangle of $\operatorname{carr}(P)$ affinely onto the corresponding triangle of $\operatorname{carr}(Q)$.

We work only with situations where $P$ and $Q$ are univalent, and in this case $F: P \longrightarrow Q$ is a piecewise affine homeomorphism. We will discuss the distortion of such mappings after the next section.

6.2. Quasiconformal Mappings. Quasiconformal mapping is a beautiful, rich, and involved subject. Fortunately, our mappings are very straightforward, and though we will use quite deep results, they are also quite intuitive and visual. So 
for us, this is a handy collection of tools, and we must refer the reader to the standard source, Lehto and Virtanen [16], for details.

An orientation-preserving homeomorphism $g: \Omega_{1} \longrightarrow \Omega_{2}$ between open sets in the plane is said to be quasiconformal ( $\kappa$-quasiconformal or $\kappa$-qc) if there is some constant $\kappa \geq 1$ so that

$$
\limsup _{r \rightarrow 0}\left\{\frac{\max _{\theta}\left\{\left|g\left(a+r e^{i \theta}\right)-g(a)\right|\right\}}{\min _{\theta}\left\{\left|g\left(a+r e^{i \theta}\right)-g(a)\right|\right\}}\right\} \leq \kappa, \text { for all } a \in \Omega_{1} .
$$

This is the most geometric among many formulations of quasiconformality and illustrates the essential feature of these mappings: namely, they act with bounded distortion on infinitesimal discs. They generalize the notion of conformal (i.e., analytic) functions; in fact, $g$ is analytic if and only if it is 1-qc. We gather the following facts:

(a) If $\Omega_{1}$ and $\Omega_{2}$ are euclidean triangles with angles bounded below by $\beta>0$ and if $g: \Omega_{1} \longrightarrow \Omega_{2}$ is affine, then $g$ is $\kappa$-qc for a constant $\kappa \geq 1$ depending only on $\beta$.

(b) If $g$ satisfies (6.1) for all $a \in \Omega_{1}$ off a set having Lebesgue area measure zero, and the left side of (6.1) is finite for the remaining $a$, then $g$ is $\kappa$-qc on all of $\Omega_{1}$.

(c) If $g$ is $\kappa$-qc, then $g^{-1}$ is $\kappa$-qc.

(d) There is no quasiconformal mapping of the plane $\mathbf{C}$ onto a proper subset of the plane. (Liouville's Theorem.)

(e) A homeomorphism of the plane to itself is quasiconformal if it satisfies the local distortion condition of (6.1) for some $\kappa \geq 1$. However, this has an important global distortion consequence, referred to as quasisymmetry.

Definition 6.1. An open set $\Omega$ in the plane is said to be $\sigma$-almost round, $\sigma \geq 1$, if the ratio $R / r<\sigma$, where $R$ is the radius of the smallest disc containing $\Omega$ and $r$ is the radius of the largest disc contained in $\Omega$.

Let $g$ be a $\kappa$-qc mapping of $\mathbf{C}$ (onto $\mathbf{C}$ ). If $\Omega$ is an open set which is $\sigma$-almost round, some $\sigma>1$, then $g(\Omega)$ is $\sigma^{\prime}$-almost round, where $\sigma^{\prime}$ depends only on $\sigma$ and $\kappa$ [17, Thm. 2.4].

(f) If $g$ is $\kappa$-qc and $h$ is $\kappa^{\prime}$-qc, then $g \circ h$ is $\kappa \kappa^{\prime}$-qc (where defined). In particular, pre- or post-composition with an analytic function preserves $\kappa$-quasiconformality.

(g) If $\left\{g_{n}\right\}$ is a sequence of $\kappa$-qc mappings of a domain $\Omega$ into the plane, then there exists a subsequence $\left\{g_{n_{j}}\right\}$ which converges uniformly on compacta to a function $g$ from $\Omega$ into the extended complex plane satisfying one of the following: (a) $g$ is constant, (b) $g$ takes two values, or (c) $g$ is a $\kappa$-qc map of $\Omega$ onto a domain $\Omega^{\prime}$. (Normal families.)

(h) A ring domain refers to an open topological annulus in the plane (or on a Riemann surface). For each ring domain $R$, there exists a conformal homeomorphism $\phi$ mapping $R$ to an annulus $A=\left\{r_{1}<|z|<r_{2}\right\}$ for some $0 \leq r_{1}<r_{2} \leq \infty$. If $r_{1}>0$ and $r_{2}<\infty$, then the ratio $r_{2} / r_{1}$ is uniquely determined and the modulus of $R$ is defined as $\operatorname{Mod}(R)=\log \left(r_{2} / r_{1}\right)$. If $r_{1}=0$, then $R$ is conformally a punctured disc if $r_{2}<\infty$ or a punctured plane if $r_{2}=\infty$; write $\operatorname{Mod}(R)=\infty$ in either case.

Let $\Omega$ be a ring domain and let $\left\{R_{j}\right\}$ be a collection (finite or infinite) of ring domains in $\Omega$, each separating the two boundary components of $\Omega$. If the $R_{j}$ are mutually disjoint, then $\operatorname{Mod}(\Omega) \geq \sum_{j} \operatorname{Mod}\left(R_{j}\right)$. In particular, suppose $\Omega$ is an open disc of (euclidean) radius $\rho_{2}$ from which a closed disc of radius $\rho_{1}$ has been 
removed. Then one can show that $\operatorname{Mod}(\Omega) \leq \log \left(\rho_{2} / \rho_{1}\right)$. With the inequality above, we obtain

$$
\rho_{1} \leq \rho_{2} \exp \left(-\sum_{j} \operatorname{Mod}\left(R_{j}\right)\right) .
$$

(i) If $R$ is a ring domain and $g: R \longrightarrow \mathbf{C}$ is $\kappa$-qc, then

$$
(1 / \kappa) \operatorname{Mod}(R) \leq \operatorname{Mod}(g(R)) \leq(\kappa) \operatorname{Mod}(R) .
$$

This quantifies the "bounded distortion" of quasiconformal maps on ring domains.

6.3. Convergence of Circle Packing Maps. The quasiconformal distortion of circle packing maps is of prime importance in our work. Let $F: P \longrightarrow Q$ be a circle packing map. In general terms, if a triangle in $\operatorname{carr}(P)$ and its counterpart in $\operatorname{carr}(Q)$ are approximately similar, then the distortion of $F$ on that face will be small.

Bounds on distortion come from the elementary but very useful Ring Lemma of Rodin/Sullivan [19]. All our complexes will have bounded degree, $\operatorname{deg}(S)<\infty$, meaning that $N=\operatorname{deg}(S)$ is an upper bound on the number of edges from any one vertex of $S$. Thus, in a packing for $S$, no circle has more than $N$ neighbors, and if the packing is univalent, then these have mutually disjoint interiors. In this case, the Ring Lemma implies a positive lower bound $\beta$ on the angles of (interior) triangles of the carrier, with $\beta$ depending only on $N$. This bound holds in both $P$ and $Q$, implying that corresponding triangles of their carriers are boundedly similar. Using result (a) of $\S 6.2$, we obtain the important

Lemma 6.2. Let $S$ be a complex of degree $N<\infty$. There exists a constant $\kappa>1$ depending only on $N$ so that if $P$ and $Q$ are any two univalent circle packings for $S$ and $F: P \longrightarrow Q$ is the circle packing map, then $F$ is $\kappa$-quasiconformal on the interior faces of carr $P$.

We will want more than bounded distortion - ultimately we want analyticity in the limit. There is a metatheorem in circle packing: the greater the combinatorial depth of a face in $S$, the less the distortion of $F$ on that face. The result we use is due to Z-X. He and Oded Schramm [10]; we have modified the statement to suit our purposes and notations and have extended their proof slightly to accommodate variable regions:

Theorem 6.3 (He/Schramm [10]). Let $\left\{D_{n}\right\}$ and $\left\{\widetilde{D}_{n}\right\}$ be sequences of Jordan domains in $\mathbf{C}$ (i.e., interiors of Jordan curves) whose closures converge to the closure of Jordan domains $D$ and $\widetilde{D}$, respectively, in the Hausdorff metric on compact sets. Let $p_{0}$ be a point of $D$. For each $n$, let $P^{n}$ be a univalent circle packing lying in $D_{n}$, let $\widetilde{P}^{n}$ be a combinatorially equivalent univalent circle packing lying in $\widetilde{D}_{n}$, and let $F_{n}: P^{n} \longrightarrow \widetilde{P}^{n}$ be the circle packing map.

Let $\delta_{n}$ be a sequence of positive numbers, tending to zero. Assume that the radii of the circles of $P^{n}$ are less than $\delta_{n}$, that each boundary circle of $P^{n}$ is within distance $\delta_{n}$ of $\partial D_{n}$, and that each boundary circle of $\widetilde{P}^{n}$ is within distance $\delta_{n}$ of $\partial \widetilde{D}_{n}$. Suppose that $p_{0} \in$ carr $P^{n}$ and the images $F_{n}\left(p_{0}\right)$ lie in a compact subset of $\widetilde{D}$. Then there exists a subsequence $\left\{F_{n_{j}}\right\}$ which converges uniformly on compact subsets of $D$ to a conformal homeomorphism $F: D \longrightarrow \widetilde{D}$. 
One can also show that if for each $n=n_{j}, r_{n}$ denotes the radius of the circle of $P^{n}$ closest to $p_{0}$ and $R_{n}$ denotes the radius of the circle of $\widetilde{P}^{n}$ closest to $\tilde{p}_{0}$, then $R_{n} / r_{n} \longrightarrow\left|F^{\prime}\left(p_{0}\right)\right|$ as $n=n_{j} \rightarrow \infty$.

\section{The Discrete Construction}

We now build a pentagonal tiling for $K$ using circle packing. There are many ways to do this, but we choose a particularly simple approach to illustrate how closely the "discrete" construction parallels the conformal one.

First, $K$ must be modified to a triangulation. We use a simple face-center triangulation; that is, add a vertex to each face of $K$ and connect it to the face's corners. The result is an infinite simplicial complex $S$ triangulating an open topological disc. It should cause no confusion to identify the 1 -skeleton of $K$ as a subgraph of the 1-skeleton of $S$.

Each pentagonal face $f$ of $K$ is composed of five triangular faces of $S$ (namely, the "star" of the central vertex for $f$ ). Such a 5 -flower gives the packing $P_{f}$ of Figure 8 , where we have set the boundary radii to $1 / 2$ and computed the central radius as $r_{c} \approx 0.35065$. The carrier of $P_{f}$ is a regular euclidean unit pentagon; thus the radii of $P_{f}$ impose the geometry of a regular pentagon on $f$. (We will see the regular unit pentagon often, so we denote it by $\mathfrak{P}$.)

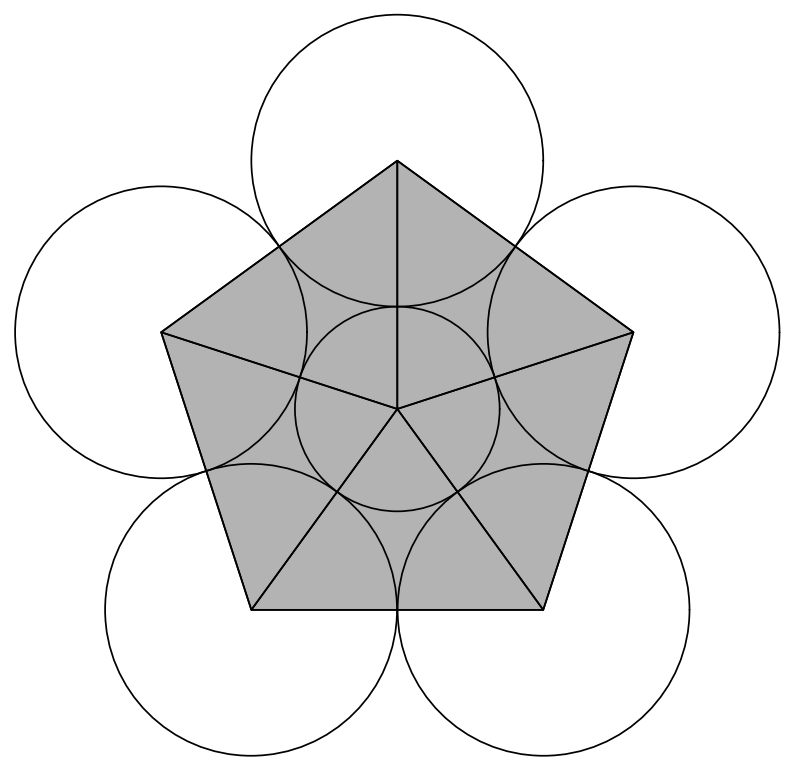

Figure 8. Packing an individual face.

Such individual face packings are our fundamental building blocks. We paste them together in the pattern prescribed by $K$ much as we have pasted faces before: whenever $f_{1}, f_{2}$ are contiguous faces of $K$, the two circles of $P_{f_{1}}, P_{f_{2}}$ associated with their common edge are identified. Equivalently, one can generate a label $\mathcal{R}$ for $S$ with radius $1 / 2$ for every vertex of $K$ and radius $r_{c}$ for the center vertex of each face. These radii then impose, face by face, precisely the familiar piecewise affine structure on $K$. 
The reader will note, of course, that $\mathcal{R}$ is not a packing label for $S$ because the packing conditions fail at the vertices of $K$. At a vertex where three (resp. four) pentagons come together, the degree is 6 (resp. 8). That means, the circle of radius $1 / 2$ for that vertex has 6 (resp. 8) neighbors each of radius $r_{c}$. This doesn't work in the plane; in fact, a computation shows that the so-called "angle sum" at this vertex will be $9 \pi / 5$ (resp. $12 \pi / 5$ ). In other words, these vertices have the "cone" angles that are already familiar from the affine structure on $K$.

There is, however, a univalent circle packing for $S$; namely, the maximal circle packing $\mathcal{P}_{S}$. No confusion should result if we call this $\mathcal{P}_{K}$ instead. There are various "type conditions" one can apply in hopes of showing whether $\mathcal{P}_{K}$ lies in the euclidean or hyperbolic plane. In this instance, we will show shortly that $\mathcal{P}_{K}$ is euclidean. In agreement with previous normalizations, we will place the center circle for the base face $f_{0}$ at the origin and the center of the circle for vertex $v_{0}$ at $z=i$. This maximal packing is pictured in Figure 2 .

The transition from the label $\mathcal{R}$ to the packing label $\mathcal{R}_{S}$ for $\mathcal{P}_{K}$ is very much in the spirit of the adjustments associated with the conformal mapping $\Phi$ described earlier. The affine structure is not globally flat, and the Riemann Mapping Theorem tells us how to adjust the surface to place it in the plane while maintaining conformality locally. Likewise, our label $\mathcal{R}$ is not globally flat (i.e., these circles just don't fit together in the plane), but our Discrete Riemann Mapping Theorem tells us how to adjust the radii to pack in the plane while maintaining the tangency relationships locally.

Note that the packing $\mathcal{P}_{K}$ also provides us with a pentagonal tiling having the combinatorics of $K$. Connecting the centers of tangent shaded circles in Figure 2 gives a pleasing embedding of $K$, though the edges are euclidean segments rather than the analytic arcs of the actual conformal tiling.

In summary, we moved from the combinatorics of $K$ to a pentagonal tiling of the plane in a fashion very much in parallel with our earlier development.

7.1. Practicum. On the practical side, the discrete approach with circle packing gives a very concrete feel to the construction, since one can implement it with the aid of software and watch the approximate tiling emerge step by step. There is a generic way to carry this out: Choose $\left\{S_{n}: n=0,1, \cdots\right\}$ to be a nested sequence of finite simply connected subcomplexes of $S$ containing $f_{0}$ and exhausting $S$. Each $S_{n}$ has a maximal packing in the unit disc by the Discrete Riemann Mapping Theorem. Apply a Möbius transformation which puts the center circle for $f_{0}$ at the origin and the circle for $v_{0}$ at $z=i$; let the resulting (euclidean) packing be denoted $\mathcal{P}_{n}$ with packing label $\mathcal{R}_{n}$. As $n$ goes to infinity the labels $\mathcal{R}_{n}$ converge numerically to the maximal packing label $\mathcal{R}_{S}$ (normalized), and in particular, the circles of the packings $\mathcal{P}_{n}$ converge to the corresponding circles in $\mathcal{P}_{K}$. There is a known algorithm for numerically approximating each $\mathcal{R}_{n}$ (and it is quite efficient), and from that one can lay out an approximation to $\mathcal{P}_{n}$. In a very practical sense, then, one has algorithms for generating maximal circle packings.

Returning to $K$, we will modify this procedure slightly, since there is a more natural way to proceed. First, we choose the sequence $\left\{S_{n}\right\}$ to be the face-center triangulations of the $n$-stage aggregate faces $f_{0}^{(n)}$ (i.e., of $K_{n}$ ). Second, in place of the maximal packings, we compute instead the univalent packing whose carrier is a regular pentagon; the normalization is the same. 
The first three stages, $\mathcal{P}_{0}, \mathcal{P}_{1}, \mathcal{P}_{2}$, are shown (in different scales) in Figure 9 . The embeddings they yield for $K_{0}, K_{1}, K_{2}$ (also shown) are actually the ones we used in Figure 4. The limit, as described above, of the normalized packings $\mathcal{P}_{n}$ is the infinite packing suggested in Figure 2.

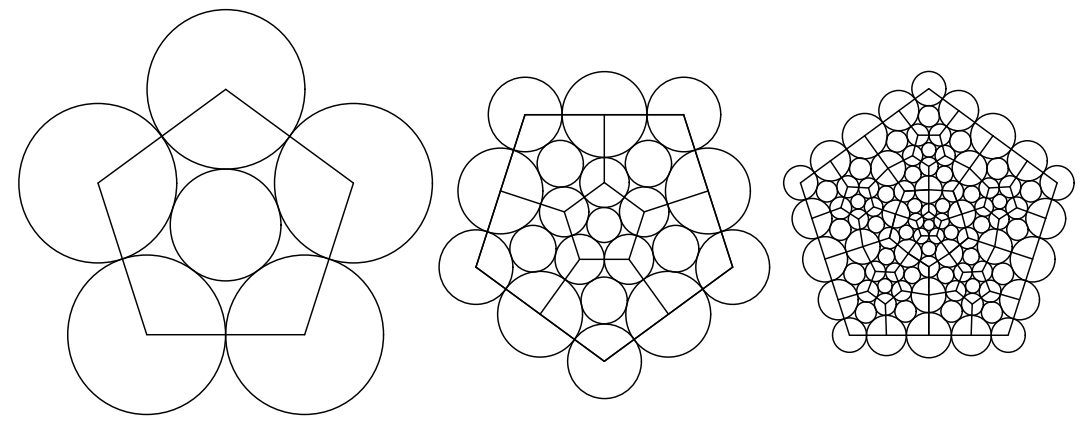

Figure 9. The first three stages via packings.

7.2. The Type Problem. We verify our claim that the maximal packing $\mathcal{P}_{K}$ for $S$ lives in the euclidean rather than the hyperbolic plane.

One method is to compare the circle packing tiling to our conformal tiling. In the affine structure on $K$, decompose each regular pentagonal face into five isosceles triangles by drawing edges from its center to its corners. This triangulation of $K$ has the same combinatorics as $S$, so one can define a piecewise affine simplicial map $h: \operatorname{carr}\left(\mathcal{P}_{K}\right) \longrightarrow K$; the uniform lower bounds on the triangles in domain and range implies that $h$ is $k$-qc on each face for some $k>1$. Since $\Phi: K \longrightarrow \mathbf{C}$ is conformal, $\Phi \circ h: \operatorname{carr}\left(\mathcal{P}_{K}\right) \longrightarrow \mathbf{C}$ is $k$-qc. By Liouville's Theorem, $\operatorname{carr}\left(\mathcal{P}_{K}\right)=\mathbf{C}$. Note that $h$ maps the union of the five triangles of $\operatorname{carr}\left(\mathcal{P}_{K}\right)$ associated with each face $f$ of $K$ one-to-one onto the associated tile $\tau \in \mathcal{T}$.

We would prefer a method more intrinsic to circle packing. The following argument is a step in this direction, though it still relies on the reflective nature of the combinatorics.

Let us establish notation. For $n \geq 1$ let $s_{n}$ denote the face-center triangulation of the $(n)$-stage pentagonal subdivision of a combinatorial pentagon (i.e., of $K_{n}$ ). One can show that there exists a univalent circle packing $Q_{n}$ of $s_{n}$ with $\operatorname{carr}\left(Q_{n}\right)=\mathfrak{P}$; packings $Q_{2}$ and $Q_{4}$ are illustrated in Figure 10.

For each face $f$ of $K$ and each $n \geq 1$, let $\mathcal{P}_{f}^{(n)}$ be circles of $\mathcal{P}_{K}$ associated with the $n$-stage aggregate face $f^{(n)}$. For each $f$ this is a circle packing with complex $s_{n}$. Let $F_{f, n}: \mathcal{P}_{f}^{(n)} \longrightarrow Q_{n}$ be the circle packing map.

Let $f_{1}, \cdots, f_{5}$ be the five faces of $K$ surrounding the base face $f_{0}$ and denote their union by $R$. Now fix $n$. Since each face is a regular unit pentagon in the affine structure of $K$, we can lift $Q_{n}$ isometrically from $\mathfrak{P}$ to each face. Because of the dihedral symmetry of $s_{n}$, and hence $Q_{n}$, the packings lifted to any two contiguous faces will be compatible along the circles of their shared edge - the circles occur with the same radii in the same order. The result is a packing $Q_{R}^{n}$ of $R$. It is extremely important to recognize the role that symmetry in the combinatorics plays in permitting this pasting. (We have Walter Parry to thank for pointing this out to us.) 

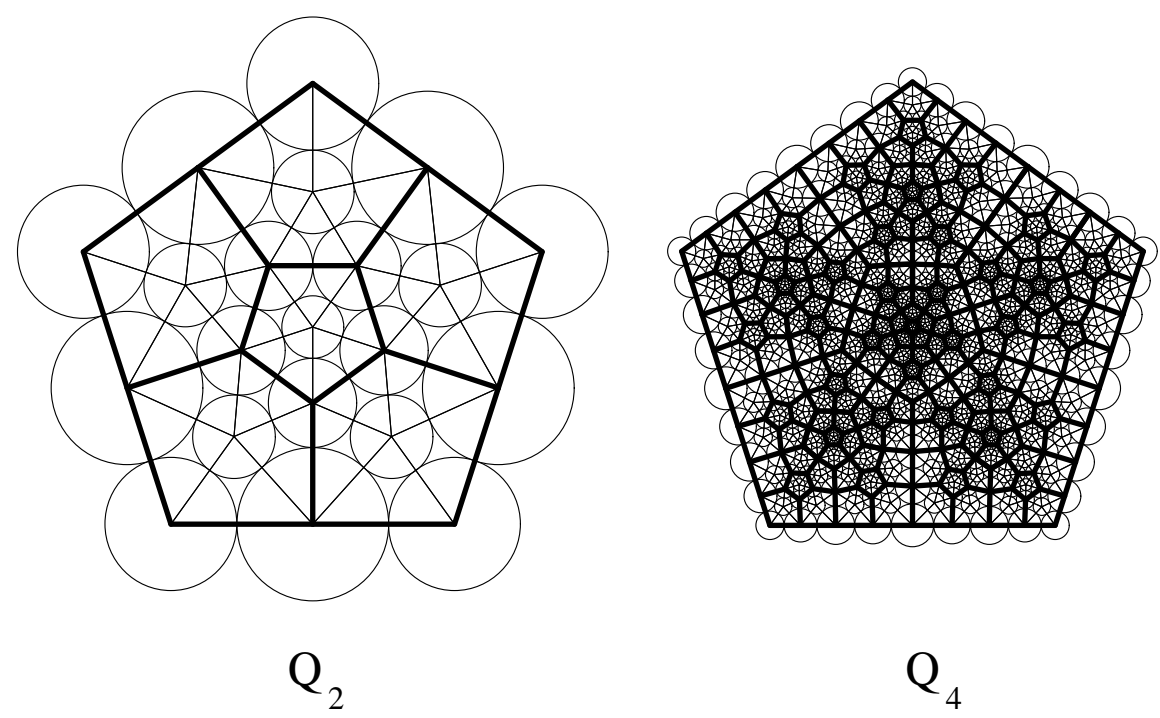

FiguRE 10. Refining by subdivision.

Over in $\mathcal{P}_{K}$, let $P_{R}^{n}$ denote the circle packing associated with the union of the five aggregate faces $f_{j}^{(n)}, j=1, \cdots, 5$, and write $C_{n}$ for $\operatorname{carr}\left(P_{R}^{n}\right)$. The circle packings $P_{R}^{n}$ and $Q_{R}^{n}$ have the same combinatorics, and the circle packing map

$$
G_{n}: \operatorname{carr}\left(P_{R}^{n}\right)=C_{n} \longrightarrow \operatorname{carr}\left(Q_{R}^{n}\right)=R
$$

is $\kappa$-qc for some $\kappa \geq 1$ by Lemma 6.2 .

The ring $R$, a topological annulus, inherits an affine and a conformal structure from $K$, hence a modulus, $0<\operatorname{Mod}(R)<\infty$ (which can actually be estimated from the tiling constant $a$ ). By (7.1) and (6.3),

$$
(1 / \kappa) \operatorname{Mod}(R)<\operatorname{Mod}\left(C_{n}\right)<\kappa \operatorname{Mod}(R), n \geq 1 .
$$

The collection $\left\{C_{n}: n=1, \cdots\right\}$ is a nested collection of rings with mutually disjoint interiors and separating the circle at the center of the base face $f_{0}$ from infinity. By (7.2) and (6.2), this implies that $\operatorname{carr}\left(\mathcal{P}_{K}\right)$ must be the euclidean plane.

\section{Approximation of Tile Shapes}

We want more out of our circle packing; namely, approximations of actual conformal tile shapes in $\mathcal{T}$. The packing $\mathcal{P}_{K}$ seems at first to be far too "coarse" - each face, defined by just six circles, is polygonal. It is clear that "finer" circle packings of the faces are needed. Thanks to the internal symmetries of $K$, however, $\mathcal{P}_{K}$ already contains these. Using aggregates of tiles, we find that all the conformal information on tiles may be obtained asymptotically from $\mathcal{P}_{K}$.

As $v_{0}$ has been the corner of $f_{0}$ used for normalization, so we designate a corner vertex $v_{n}$ of each aggregate $f_{0}^{(n)}, n \geq 1$. (The choice is immaterial due to rotational symmetry.) Define $\mathcal{P}^{(n)}=\lambda_{n} \mathcal{P}_{K}$, with $\lambda_{n} \in \mathbf{C}$ chosen to center $v_{n}$ at $z=i$. We continue to use $\mathcal{P}_{f}^{(n)}$ to denote the circle packing associated with the $n$-stage aggregate of face $f$ (but now $\mathcal{P}_{f}^{(n)}$ is part of the scaled packing $\mathcal{P}^{(n)}$ ). 
Fix $n$. For each $f \in K$, identify $\operatorname{carr}\left(\mathcal{P}_{f}^{(n)}\right)$ with $\operatorname{carr}\left(Q_{n}\right)$ via the circle packing map; since $\operatorname{carr}\left(Q_{n}\right)$ is a regular unit pentagon, we may then identify it isometrically with the face $f$ of $K$ (in its affine structure). Ensure that the map for $f_{0}$ carries corner $v_{n}$ to the vertex $v_{0}$ of $K$. The various maps are well defined on edges shared by (aggregate) faces because of their dihedral symmetry. The combinatorics of the aggregate faces are the same as those of $K$, so we arrive at a homeomorphism $G_{n}: \mathbf{C} \longrightarrow K$, and composing with $\Phi$ we have the final homeomorphism $H_{n}=$ $\Phi \circ G_{n}: \mathbf{C} \longrightarrow \mathbf{C}$.

By Lemma 6.2 and the analyticity of $\Phi$, each $H_{n}$ is $\kappa$-qc for some $\kappa>1$, and our normalizations imply

$$
H_{n}(0)=0, \quad H_{n}(i)=i, \quad H_{n}(\infty)=\infty .
$$

Observe that for each $n$ and each $f$,

$$
H_{n}\left(\operatorname{carr}\left(\mathcal{P}_{f}^{(n)}\right)\right)=\tau,
$$

where $\tau$ is the conformal tile associated with $f$.

By normal families, there exists a convergent subsequence $\left\{H_{n_{j}}\right\}$. (For convenience and without loss of generality, assume this is the full sequence.) The limit function $H$ is, by (8.1), a $\kappa$-qc homeomorphism of the plane fixing $0, i$, and $\infty$.

Claim. The function $H$ is the identity function.

Proof of Claim. It is enough to show that $H$ is 1-qc, hence an analytic function, on the Riemann sphere which fixes three points. We can concentrate on tile interiors, since their boundaries have area zero.

In the following, whenever we use $\tau$ for a tile, $f$ will denote the corresponding face. For each $\tau$, write $D_{n}^{\tau}=\operatorname{int}\left(\operatorname{carr}\left(\mathcal{P}_{f}^{(n)}\right)\right)$. These domains have closures which converge (in the Hausdorff metric) to the closure of the Jordan domain $D^{\tau}=$ $H^{-1}(\operatorname{int}(\tau))$. It suffices to show that $H: D^{\tau} \longrightarrow \tau$ is analytic, and for this we use the Theorem of He/Schramm.

First, an observation about conformal moduli of certain ring domains within our tiling. Each tile $\tau$ is surrounded by a closed chain consisting of the five tiles sharing edges with $\tau$ and from zero to three additional tiles sharing vertices, the number depending on the tile corner angles (every tile has 0,2 , or 3 right angles, the other angles being $3 \pi / 5$; see Figure 3 ). The interior of their union forms an open conformal annulus separating $\tau$ from infinity. Within that is a smaller annulus which we denote $A_{\tau}$ obtained as follows: Subdivide each of the tiles neighboring $\tau$ using the pentagonal subdivision rule into six conformally regular pentagons. $A_{\tau}$ is the ring domain consisting of the "outer" smaller tiles in the neighbors of $\tau$ - those separated from $\tau$. Two examples are illustrated in Figure $11 ; \tau$ is the dark center tile, the lightly shaded ones form $A_{\tau}$. There are only finitely many conformally distinct patterns which can occur, so there exists a bound $\beta>0$ with

$$
\beta<\operatorname{Mod}\left(A_{\tau}\right), \tau \in \mathcal{T} .
$$

Moving now to $\mathcal{P}_{K}$, note that analogous patterns occur in its faces at every aggregation level. For a $j$-stage aggregate face $f^{(j)}$, the corresponding annulus is formed by a union of $(j-1)$-stage aggregates. Let $R_{\tau}^{(j)}$ denote the interior of the carrier of this annulus. By the same arguments used earlier to establish the type 

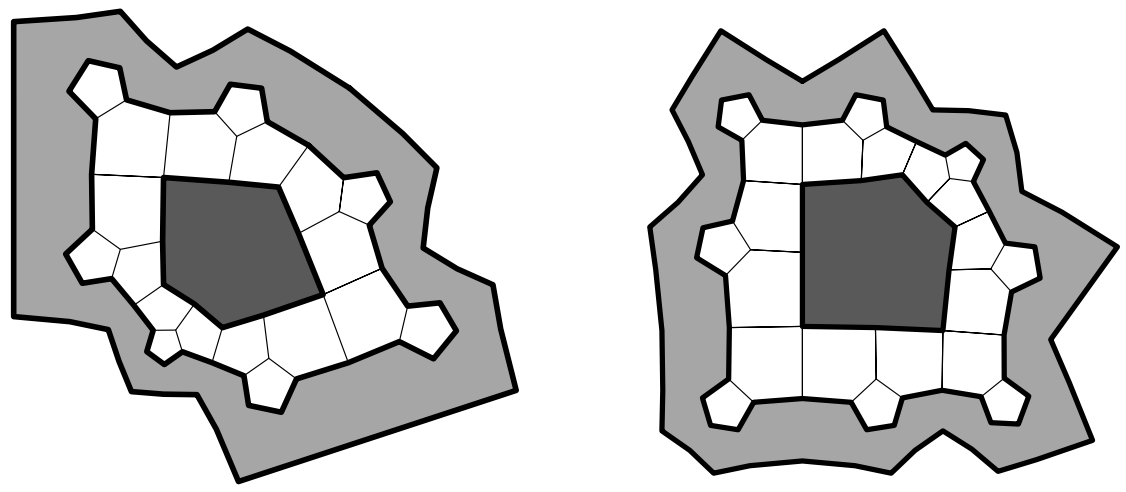

Figure 11. Typical conformal annuli $A_{\tau}$.

of $\mathcal{P}_{K}, R_{\tau}^{(j)}$ is $\kappa$-qc equivalent to $A_{\tau}$, and therefore by (8.3) satisfies

$$
(1 / \kappa) \beta<\operatorname{Mod}\left(R_{\tau}^{(j)}\right), \tau \in \mathcal{T}, j \geq 1
$$

Now, fix a tile $\tau$; write $D_{n}=D_{n}^{\tau}$ and $D=D^{\tau}$. Our first task is to prove that the circles of $\mathcal{P}_{f}^{(n)}$ go uniformly to zero in radius as $n$ goes to infinity (something which seems quite evident from the pictures). As a preliminary, the convergence of the $H_{n}$ implies existence of a real number $\rho>0$ so that

$$
R_{\tau}^{(n)} \subset\{|z|<\rho\}, n=1,2, \cdots .
$$

Given $n$, let $c$ denote a circle of $P_{f}^{(n)}$. This is associated with a vertex belonging to some face $g$ of $K$. Identify this as $g=g_{0}$, thinking of it as a 0 -stage aggregate. Inductively define $g_{j}$ to be the unique face of $K$ such that $g_{j}^{(j)} \supset g_{j-1}^{(j-1)}, j=1, \cdots, n$. In other words, the $g_{j}$ are the faces whose successive aggregates are nested starting from $g_{0}$. Observe that $g_{n}=f$. For each $j=1, \cdots, n$, let $R_{j}$ denote the ring $R_{\tau_{j}}^{(j)}$ as defined above, where $\tau_{j}$ is the tile associated with $g_{j}$; the last ring $R_{n}$ is $R_{\tau}^{(n)}$. The rather complicated definition of these rings now pays off; the reader can confirm that the $R_{j}$ are mutually disjoint. Therefore, by (8.5), we have a nested sequence of $n$ ring domains separating $c$ from $\{|z|=\rho\}$, and by (8.4) each has conformal modulus bounded below by $\beta / \kappa$. The inequality (6.2) implies that the radius of $c$ is bounded by $\rho \exp (-n \beta / \kappa)$, which goes to zero as $n$ grows.

This puts us in position to apply the He/Schramm result. For each $n$, let $P_{n}^{\prime}$ denote the circle packing $\mathcal{P}_{f}^{(n)}$ with its boundary circles removed and let $Q_{n}^{\prime}$ denote the combinatorially equivalent packing $Q_{n}$ with its boundary circles removed. The carrier of $P_{n}^{\prime}$ lies in $D_{n}$ and its boundary circles are close to $\partial D_{n}$; the $D_{n}$ converge to $D$. The arguments above regarding radii of circles in $\mathcal{P}_{f}^{(n)}$ can be modified slightly to establish a similar conclusion for circles of $Q_{n}$. Let $\widetilde{D}_{n}=\widetilde{D}=\mathfrak{P}$ for every $n$. We have confirmed the hypotheses of Theorem 6.3. Some subsequence of the circle packing maps $F_{n}: P_{n}^{\prime} \longrightarrow Q_{n}^{\prime}$ converges uniformly on compacta of $D$ to a conformal homeomorphism $F: D \longrightarrow \widetilde{D}$. If we follow $F$ by an isometric isomorphism from $\widetilde{D}=\mathfrak{P}$ to the face $f$ in the affine structure of $K$ and follow this by $\Phi$, the result is precisely the restriction of $H$ to $D=D_{t}$. The analyticity of $F$ 
therefore implies the analyticity of $H$ on the interior of $\tau$. Since $\tau$ was an arbitrary tile, our Claim is proven.

Now that we know $H$ is the identity, (8.2) implies: For each conformal tile $\tau$, the carriers of the $n$-stage aggregates for the associated face $f$ converge to $\tau$ as $n$ goes to infinity.

In hindsight, the map $H_{n}$ is roughly (though not precisely) equal to $\alpha^{n}\left(h\left(\lambda_{n} z\right)\right)$, where $h$ is the mapping defined in the beginning of $\S 7.2, \alpha$ is our self-similarity for $\mathcal{T}$, and $\lambda_{n}$ is the scaling factor normalizing $\mathcal{P}_{K}(n)$. One sees that $\sqrt[n]{\left|\lambda_{n}\right|}$ converges to $1 /|a|$, for our tiling constant $a$. Also, by extending our arguments one can prove that when faces $f_{1}$ and $f_{2}$ are contiguous across an edge $e$, then the obvious circle packing map interchanging $\mathcal{P}_{f_{1}}^{(n)}$ and $\mathcal{P}_{f_{2}}^{(n)}$ will converge to the anticonformal reflection $r_{e}$ interchanging the corresponding tiles $\tau_{1}$ and $\tau_{2}$. In particular, the ratios of the radii of corresponding circles will converge to $\left|r_{e}^{\prime}\right|$. Applying this at the central circles of the $n$-stage aggregates of $f_{0}$, one obtains estimates of the tiling constant $b$. From the packing of Figure $1, b \approx 1.2$.

There is one final detail to point out. In practice, one does not have access to the infinite packing $\mathcal{P}_{K}$. However, as we described in $\S 7$, it is approximated by the maximal packings associated with the finite complexes $K_{n}$, and these maximal packings are effectively computable. One can show that the carrier of the $n$-stage aggregate face $f^{(n)}$ within the normalized maximal packing associated with $K_{n^{2}}$ will converge to $\tau$. So tile shapes are effectively computable.

\section{Part III. Generalizations}

We have concentrated on a particular pentagonal tiling for purposes of illustration and because of its connections, via subdivision rules, to other work. The reader may see many directions for generalization. We mention a few.

As to combinatorics, there is an infinite variety of pentagonal complexes we could consider in place of $K$. There is no need, either, to restrict to pentagonal faces in $K$, and no need to have combinatoric symmetries. Our methods produce conformally regular tilings with faces having any numbers of edges and in any (planar, simply connected) pattern. To illustrate, Figure 12 involves regular triangles, rectangles, and pentagons; this happens to be associated with a subdivision rule, the "dodecahedral variant" of [12, Figure 19]. Of course, the key issue is type: does the result tile $\mathbf{C}$ or $\mathbf{D}$ ? One can apply the first argument of $\S 7.2$ to show that the type of a pattern involving a finite number of polygonal shapes is the same as that of the circle packing for its face-center triangulation.

Even for single-tile-type subdivision rules, there are many $n$-gonal tilings to consider. For dihedrally symmetric rules of bounded valence, our work generalizes directly. For instance, the rule for $n=7$ given by Figure 13(c) leads to the "septagonal" tiling of Figure 14. There are self-similarities, approximating circle packings, associated tiling constants, and so forth. (Note that the rules of Figure 13 all involve adding additional vertices to the edges to form the appropriate $n$-gons.)

When dihedral symmetry is missing, interesting new wrinkles can arise. Consider $n=6$ and the subdivision rule of Figure 13(b). One can build the associated infinite complex $K$ despite the lack of local reflectivity. In fact, the tiling for $K$ turns out to be the familiar regular hexagonal tiling from antiquity. The patterns of the subdivision rule are seen only at the various aggregation levels - the aggregates are not similar to individual tiles. Asymptotically, the aggregate faces converge 


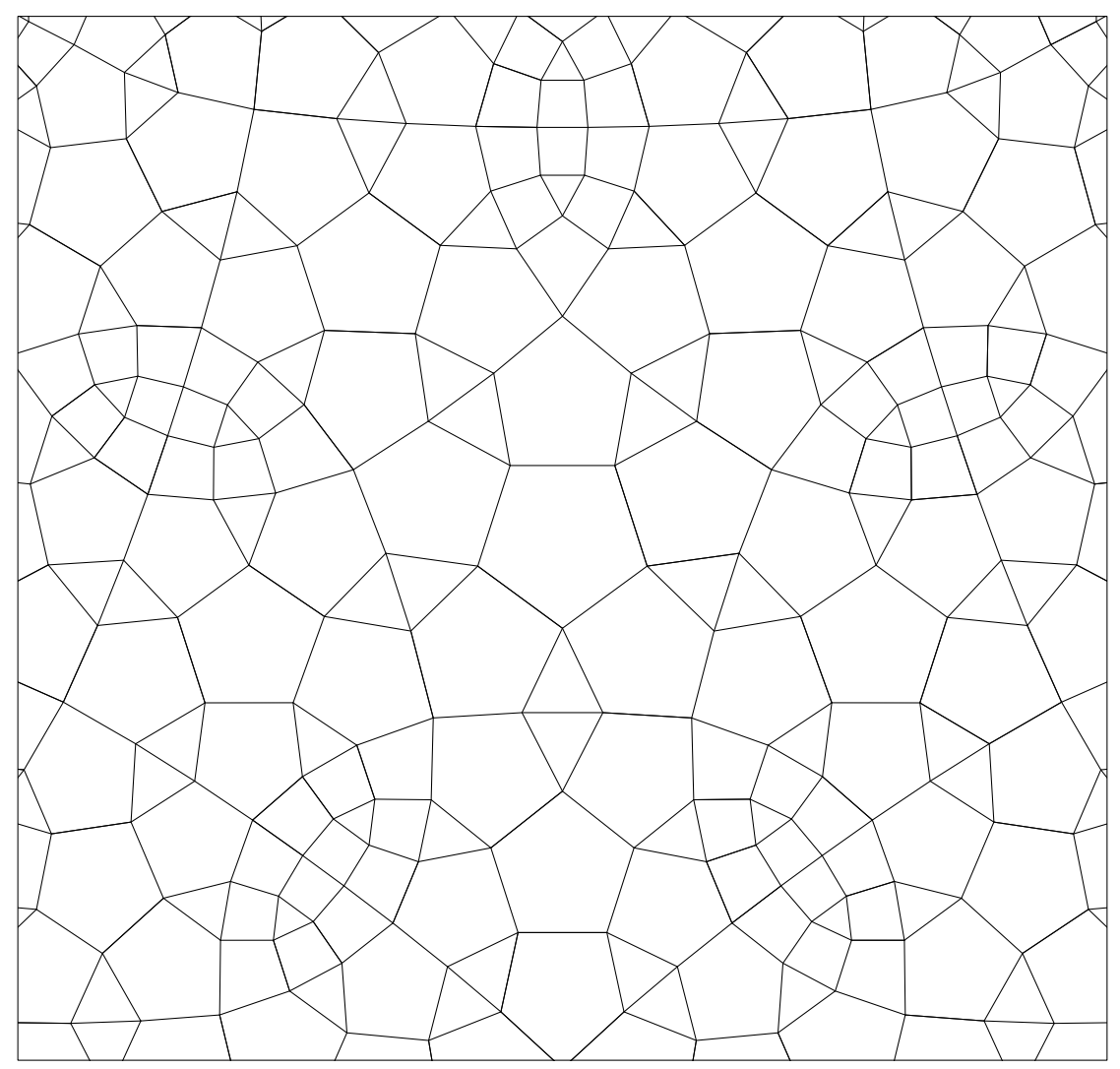

FIgURE 12. Tiling for "dodecahedral variant".

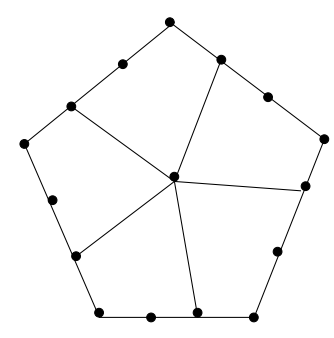

(a) $n=5$

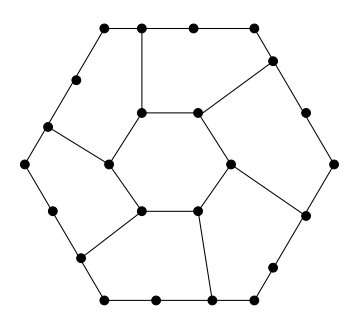

(b) $n=6$

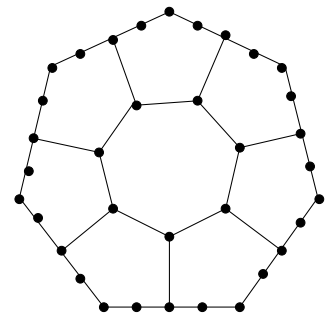

(c) $n=7$

Figure 13. Other subdivisions rules.

to the well-known "fractal map of France" shown in [12, Figure 42]. This is a Jordan region $J$ having a fractal boundary; congruent copies of $J$ will tile the plane in a hexagonal pattern, and each is a union of seven pieces similar to $J$. This emergence of an "asymptotic tiling" is quite fascinating. We actually have the same feature in our pentagonal case, though it is somewhat obscured by the 


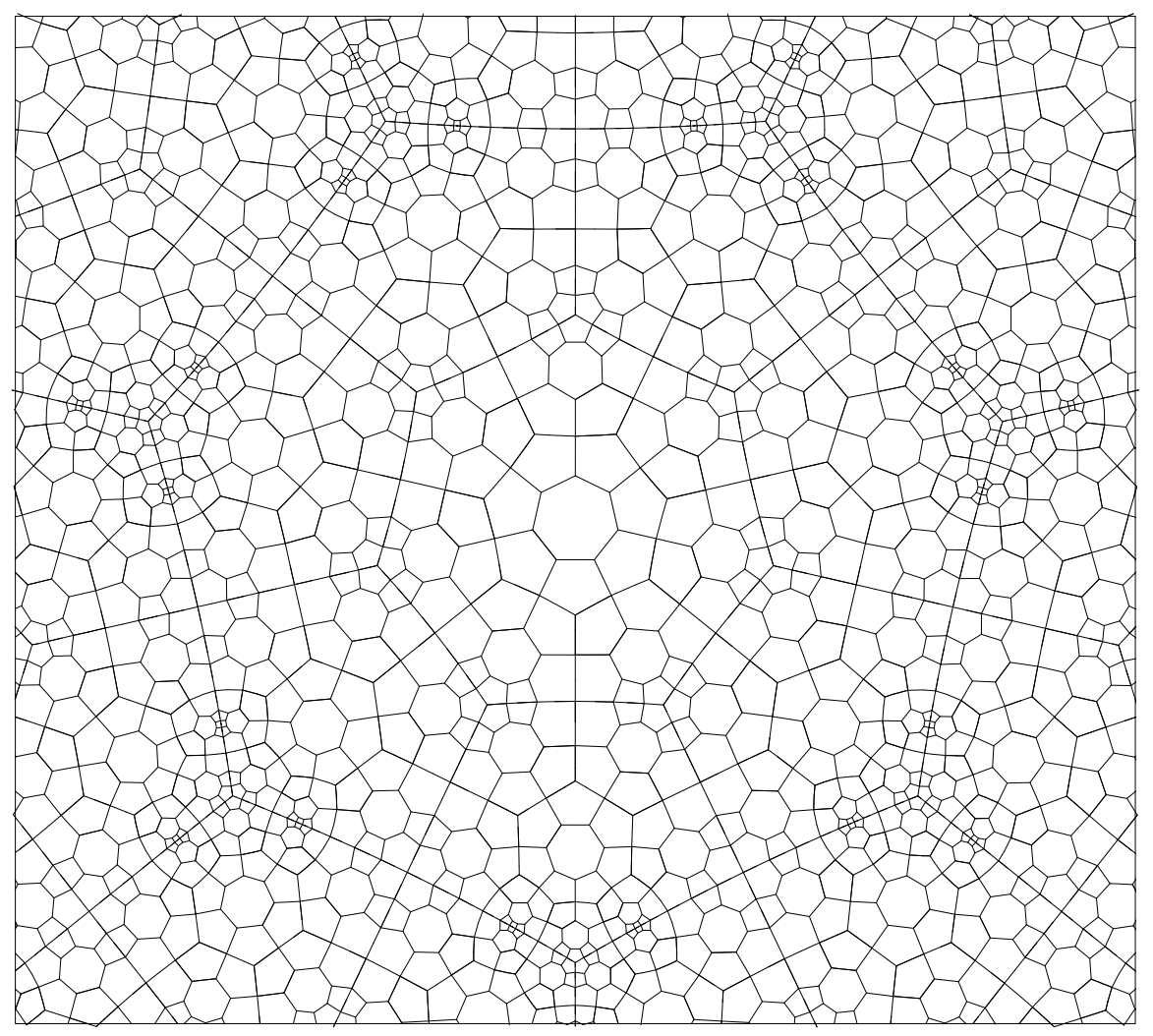

Figure 14. A septagonal tiling.

ubiquitous self-similarities. In particular, it is immaterial whether we consider tiles or aggregate tiles, and the tiles converge (in our tile families) to asymptotic shapes which themselves tile the plane (because they are actually the shapes of $\mathcal{T}$ itself).

The pentagonal subdivision rule illustrated in Figure 13(a) does not have dihedral symmetry. The associated tiling is suggested by Figure 15 . As in the $n=6$ case, the aggregate shapes (some are indicated by the darker edges) become fractal. Evidence from associated circle packings suggests that this tiling is euclidean, but there is as yet no proof.

How does one obtain tile shapes in more general situations? We could have used an alternate technique for the pentagonal tiling which would have avoided use of its global symmetries - a method we term hexagonal refinement.

Recall our need for "finer" packings of the faces than those given directly by $\mathcal{P}_{K}$. Any triangulation $S$ of a topological surface has an associated "refined" triangulation hex $(S)$ obtained by adding a vertex to the middle of each edge of $S$ and adding three edges in each face of $S$ to connect pairs of the new vertices. Thus, each triangle of $S$ is broken into four triangles in $\operatorname{hex}(S)$. The vertices of $S$ remain, with their degrees unchanged; each new (interior) vertex has degree six, hence the adjective "hexagonal". One can show that the type of the complex is unchanged, so the maximal packings of successive hex refinements provide successively finer packings associated with each face. Figure 16 illustrates the packings of $\mathfrak{P}$ obtained 


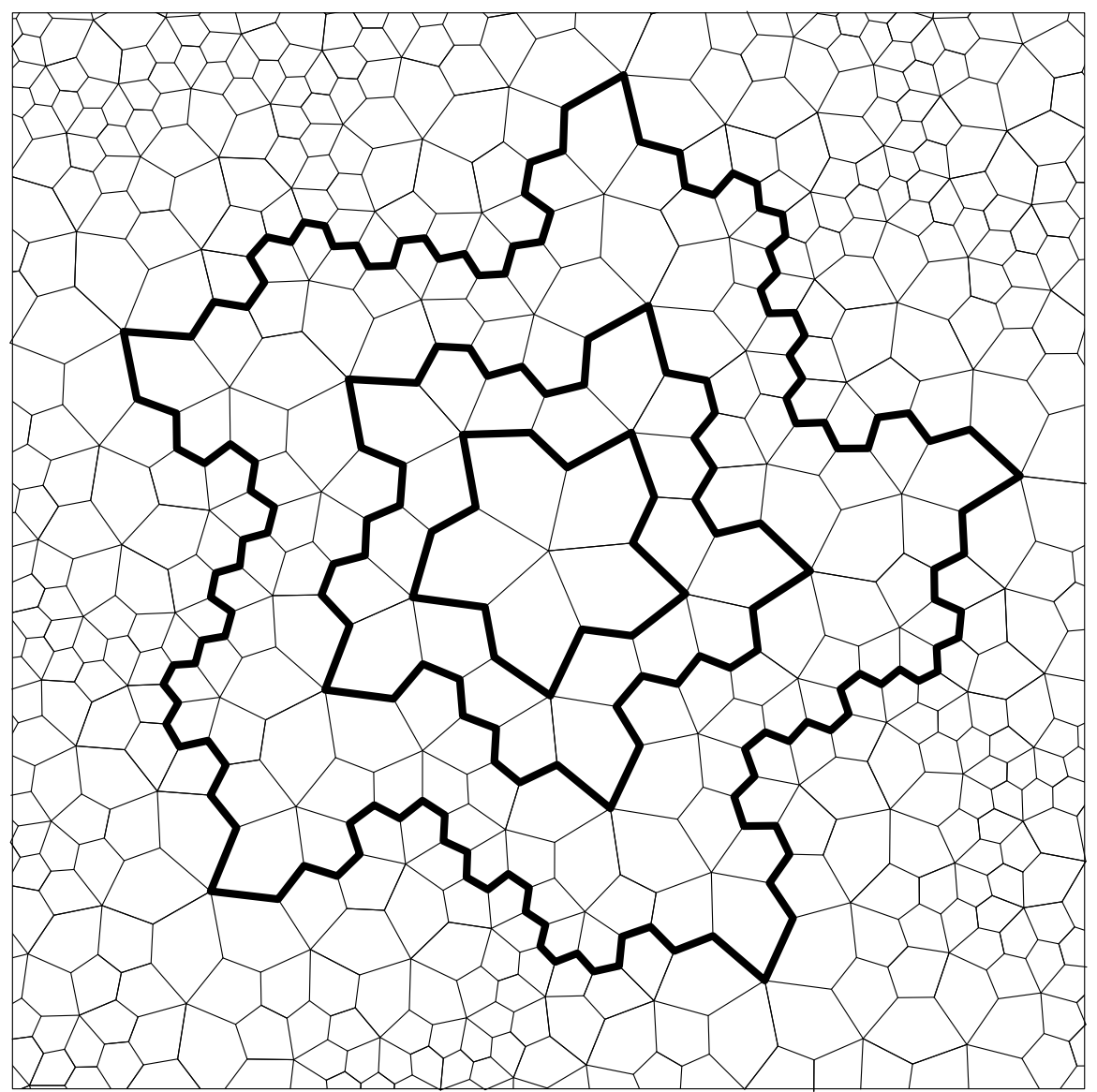

Figure 15. A "twisted" pentagonal tiling.

from the simple face-center triangulation by, respectively, 1 and 3 stages of hex refinement. These packings could have played the roles of the packings in Figure 10, with convergence questions resolved by the Hexagonal Packing Lemma of [19]. We omit the proof.

An advantage of hex refinement is that one can approximate conformally correct shapes in much more general circumstances, such as those of Figures 12 and 15. Many intriguing questions remain about such conformal tilings. We will not attempt any great detail here, but we close this subsection by warning the reader about certain subtleties (fascinating in themselves).

Consider the dodecahedral variant pattern $K$ of Figure 12, consisting of squares, triangles, and pentagons. It happens that there exist infinitely many tilings $\mathcal{T}$ of the plane by conformally regular tiles which share this same pattern (all Möbius inequivalent to one another). Here are two constructions: Give $K$ a piecewise affine structure which makes each $n$-polygon into a regular euclidean $n$-gon of unit side length; the consequent conformal structure gives a tiling $\mathcal{T}_{1}$ as described in Part I. Alternately, give $K$ a second affine structure: star each $n$-gon to break it into $n$ triangles, make each into an equilateral euclidean triangle of unit side length, 

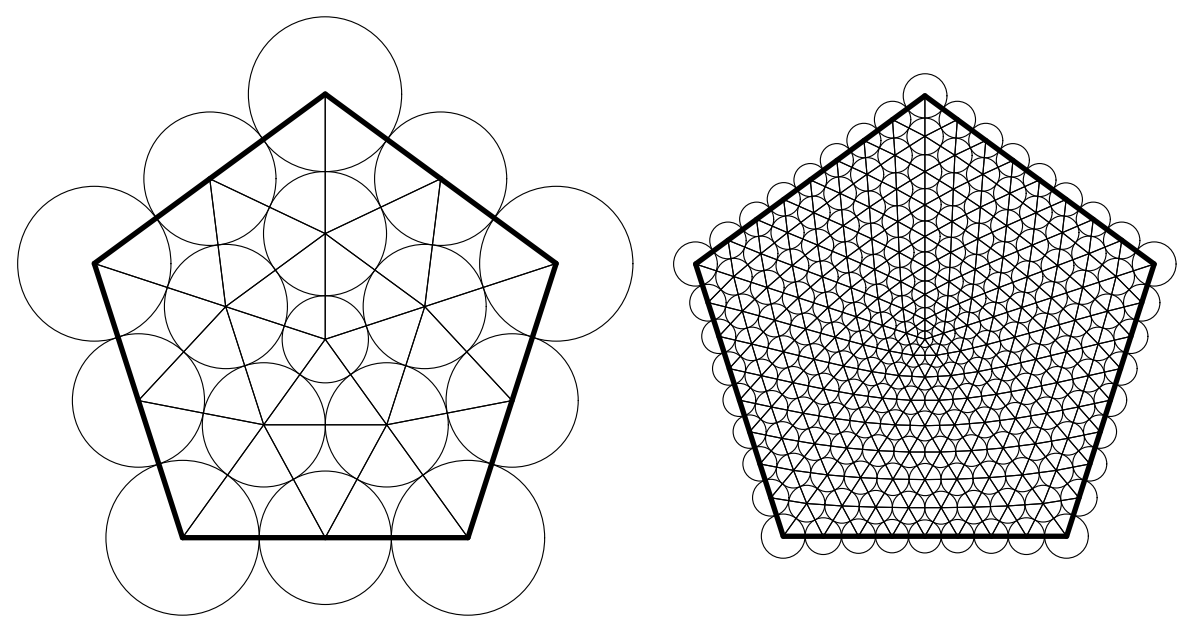

FiguRE 16. Hex refined faces.

and again map conformally to the plane. Reconstitute each face of $K$ from the associated conformal triangles, forming the second tiling $\mathcal{T}_{2}$.

Both $\mathcal{T}_{1}$ and $\mathcal{T}_{2}$ consist of conformally regular polygons in the pattern of $K$, each tile with analytic arcs as edges. Nonetheless, one can show that the union of a pentagon and a contiguous triangle from $\mathcal{T}_{1}$ will not be conformally equivalent to the union of the corresponding tiles from $\mathcal{T}_{2}$. Thus $\mathcal{T}_{1}$ and $\mathcal{T}_{2}$ are Möbius inequivalent.

A maximal circle packing can be generated from $K$ as we did in the pentagonal case, call it $\mathcal{P}_{K}$ again. This provides quasiconformal information applicable to both tilings, but its essence is the analogue of $\mathcal{T}_{2}$. The triangles used in the affine structure of $\mathcal{T}_{2}$ provide a "local reflective" structure (as in the pentagonal case); this is precisely mimicked in the circle packing structure and, moreover, is preserved under hex refinement. Indeed, one can show that the limit shapes resulting from successive hex refinement of $\mathcal{P}_{K}$ are precisely the shapes in $\mathcal{T}_{2}$. On the other hand, the three different euclidean polygons used in the construction of $\mathcal{T}_{1}$ lack local edge reflectivity, and in fact we know of no way to approximate these tile shapes.

\section{Part IV. Division Rules and Shinglings}

The pentagonal tiling in which we have invested so much effort arises in the study of "shinglings" initiated by Cannon $[7,6]$ and being pursued in work of Cannon, Floyd, and Parry and others $[14,13,12,11]$. (For convenience, we will refer to that line of work as CFP.) This topic, in turn, is motivated by questions about hyperbolic groups and their actions on 3 -manifolds.

Briefly, a finitely presented group $G$ which is word hyperbolic acts on the ideal boundary $\partial G$ of its Cayley graph. According to Thurston's Geometrization Conjecture, if the ideal boundary is topologically a 2 -sphere, then the action should be "conformal" for an appropriate conformal structure on the 2-sphere boundary. Cannon has proposed obtaining such a conformal structure using the combinatorics of shinglings that arise from the action. 
Shingles are compact connected sets and shinglings are patterns of shingles which may overlap. There is a sequence of shinglings of $\partial G$ identified with certain halfplanes of the Cayley graph of $G$. The subdivision rules tell one how to move from one shingling to the next. One hopes to use the combinatorics of these shingling patterns to extract a compatible conformal structure on $\partial G$.

In this context, our pentagonal situation is truely a "toy" problem: the subdivision rule is very simple, with a single tile type; we end up with a tiling (no overlaps); and the pattern is not, in fact, associated with a hyperbolic group action. The results are atypically clean - the central issues are somewhat confounded because $\mathcal{T}$ is such a perfectly faithful and rigid conformal representation of $K$. Nonetheless, we were confronted with the topic's fundamental obstacles, and we want to close by highlighting how these have been resolved. This is presented for the reader familiar with the work of Cannon, et al., with the expectation that alternate approaches may be useful in the broader study.

The central issue in CFP is whether the sequence of tilings generated by a subdivision rule is "conformal". This has a very precise meaning - a certain set of axioms must be verified. Subsequent work has provided various simplified/modified conditions. (Incidently, among our examples, only the "twisted pentagon" of Figure 15 remains at issue - the others are known to be conformal.) See [12] and its references.

First, we have converted the problem from "subdivision" rules to "aggregation" rules, allowing us to work with an infinite complex which contains within it every finite subdivision stage. The complex $K$ was obtained by a reflective process here; in general, multiple tile types and asymmetries add complications, including the need to look at several different infinite complexes depending on the particular "focus" tile.

Next we have added two new representations for $K$ : as carrier of a circle packing and as a conformal tiling. The combinatoric, packing, and conformal representations reflect increasing rigidity. On the other hand, each stage determines the others; results in one setting frequently (though not invariably) apply in the others. Aesthetically, one would like to answer questions using the least possible structure, but the additional resources are available if needed. The issues are rather tightly linked in our case, but let us mention them separately.

The first issue concerns the "type" of the subdivision rule, and converts here to the question of whether $\mathcal{T}$ tiles $\mathbf{C}$ or $\mathbf{D}$. We relied on the conformal rigidity of the scaling function $\alpha$. However, independent confirmation was given by showing that $\mathcal{P}_{K}$ was parabolic. Though we used the symmetries within $K$ for this, one can try some of the many type criteria established for packings (see [19, 3, 4, 18]); random walk criteria seem particularly promising (see, e.g., [9, 15]).

A directly related issue is the "expansion map" for a division rule. Combinatorially, this is the isomorphism from the subdivided complex $K^{\prime}$ to $K$. In the tiling, this led to the Möbius transformation $\alpha$, which reflects much stronger conformal rigidity than one would normally anticipate. Generally, one would expect only quasiconformal scaling which, as suggested with the map of France example earlier, might yield precise conformal information only asymptotically. In the circle packing representation, the scaling is reflected in the division-rule-based refinement process described in $\S 8$ (which approximates the conformally correct scaling factor).

CFP has established important criteria for conformality of subdivision rules in terms of uniformly "almost round" embeddings. For us, the issue is existence of an 
almost round embedding for $K$; that is, an embedding in $\mathbf{C}$ whose 2-cells are uniformly almost round in the sense of Definition 6.1. How to build such embeddings? Consider Figure 17, which displays stages in an attempt at an affine embedding of $K$. Clearly, extreme distortions will develop in later stages. (Our thanks to Scott Sheffield, via Bill Floyd, for these images.) Contrast this with our circle packing
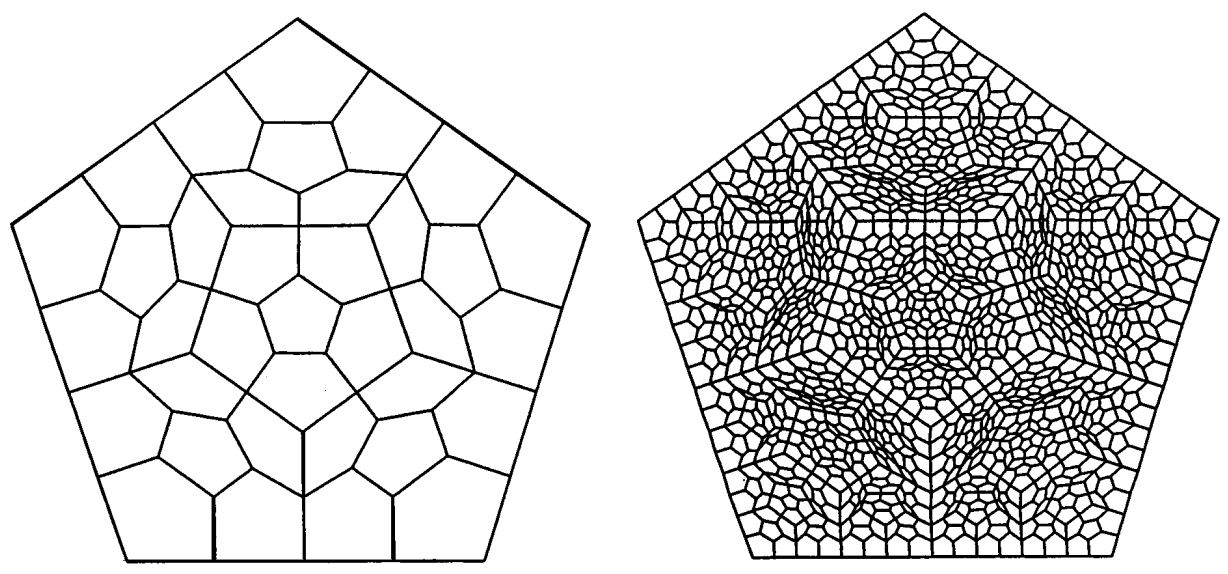

FIGURE 17. Straightline subdivision stages.

embedding, which was guaranteed to be almost round by the Rodin/Sullivan Ring Lemma. This applies in considerable generality, and an appropriate quasiconformal mapping, such as $h$ defined at the beginning of $\S 7.2$, will imply that the associated tiling is also almost round.

Finally, we mention the issue of "punctures"; is each face of $K$ "infinitely deep" in the combinatorics of $K$ ? This is confounded with the other issues, but in spirit is addressed for the packing $\mathcal{P}_{K}$ in the arguments of $\S 8$ related to Figure 11.

Though our pentagonal tiling works out perhaps too cleanly, the effort has suggested several routes for introducing classical conformal structures and their machinery into more general situations. It is clear that circle packing is potentially valuable as an intermediary, bridging the gap between the purely combinatoric and rigidly conformal natures of $K$; it also offers geometric insights of its own while providing an environment where actual experiments and computations are feasible.

\section{REFERENCES}

1. L. V. Ahlfors and L. Sario, Riemann surfaces, Princeton University Press, Princeton, New Jersey, 1960. MR 22:5729

2. A. F. Beardon, A primer on Riemann surfaces, London Math. Soc. Lecture Note Series, vol. 78, Cambridge University Press, Cambridge, 1984. MR 87h:30090

3. Alan F. Beardon and Kenneth Stephenson, The uniformization theorem for circle packings, Indiana Univ. Math. J. 39 (1990), 1383-1425. MR 92b:52038

4. _ Circle packings in different geometries, Tohoku Math. J. 43 (1991), 27-36. MR 92a:52023

5. Philip L. Bowers and Kenneth Stephenson, A branched Andreev-Thurston theorem for circle packings of the sphere, Proc. London Math. Soc. (3) 73 (1996), 185-215. MR 97d:52027

6. James Cannon, The combinatorial Riemann mapping theorem, Acta Mathematica 173 (1994), 155-234. MR 95k:30046 
7. James W. Cannon, The theory of negatively curved spaces and groups, Ergodic Theory, Symbolic Dynamics and Hyperbolic Spaces (Tim Bedford, Michael Keane, and Caroline Series, eds.), Oxford Science Publications, Oxford-New York-Tokyo, 1991 (comments on circle packing, pp. 349-351). CMP 92:02

8. Tomasz Dubejko and Kenneth Stephenson, Circle packing: experiments in discrete analytic function theory, Experimental Mathematics 4 (1995), no. 4, 307-348. MR 97f:52027

9. Zheng-Xu He and Oded Schramm, Hyperbolic and parabolic packings, Discrete Computational Geom. 14 (1995), 123-149. MR 96h:52017

10. - On the convergence of circle packings to the Riemann map, Invent. Math. 125 (1996), 285-305. MR 97i:30009

11. J. W. Cannon and E. L. Swenson, Recognizing constant curvature discrete groups in dimension 3, preprint, 1996.

12. J. W. Cannon, W. J. Floyd, and Walter Parry, On the conformal invariance of tiling-systems, preprint, 1996.

13. _ Sufficiently rich families of planar rings, preprint, 1996.

14. _ Squaring rectangles: the finite Riemann Mapping Theorem, Contemporary Math. 169 (1994), 133-212. MR 95g:20045

15. Richard Kenyon, Tilings and discrete Dirichlet problems, preprint, 1996.

16. O. Lehto and K. I. Virtanen, Quasiconformal mappings in the plane, 2nd ed., Springer-Verlag, New York, 1973. MR 49:9202

17. Olli Lehto, Univalent functions and Teichmüller spaces, Springer-Verlag, New York, 1987. MR 88f:30073

18. Gareth McCaughan, A recurrence/transience result for circle packings, Proc. Amer. Math. Soc., to appear. CMP 95:11

19. Burt Rodin and Dennis Sullivan, The convergence of circle packings to the Riemann mapping, J. Differential Geometry 26 (1987), 349-360. MR 90c:30007

20. William P. Thurston, Groups, tilings and finite state automata, The Geometry Center Research Report GCG-1 (1989), Summer 1989 AMS Colloquium Lectures.

Department of Mathematics, Florida State University, Tallahassee, Florida 323063027

E-mail address: bowers@gauss.math.fsu.edu

Department of Mathematics, University of Tennessee, Knoxville, Tennessee 37996 1300

E-mail address: kens@math.utk.edu 